\title{
Ferroelectrics: a pathway to switchable surface chemistry and catalysis
}

\author{
Arvin Kakekhani ${ }^{1,2}$, Sohrab Ismail-Beigi ${ }^{1,2,3,4}$, Eric I. Altman ${ }^{2,5, *}$ \\ ${ }^{1}$ Department of Physics, ${ }^{2}$ Center for Research on Interface Structure and Phenomena \\ (CRISP), ${ }^{3}$ Department of Mechanical Engineering and Materials Science, ${ }^{4}$ Department of \\ Applied Physics, ${ }^{5}$ Department of Chemical and Environmental Engineering, Yale \\ University, New Haven, CT 06520, USA \\ *Corresponding Author: Eric I. Altman \\ eric.altman@yale.edu
}

\begin{abstract}
It has been known for more than six decades that ferroelectricity can affect a material's surface physics and chemistry thereby potentially enhancing its catalytic properties. Ferroelectrics are a class of materials with a switchable electrical polarization that can affect surface stoichiometry and electronic structure and thus adsorption energies and modes; e.g., molecular versus dissociative. Therefore, ferroelectrics may be utilized to achieve switchable surface chemistry whereby surface properties are not fixed but can be dynamically controlled by, for example, applying an external electric field or modulating the temperature. Several important examples of applications of ferroelectric and polar materials in photocatalysis and heterogeneous catalysis are discussed. In photocatalysis, the polarization direction can control band bending at water/ferroelectric and ferroelectric/semiconductor interfaces, thereby facilitating charge separation and transfer to the electrolyte and enhancing photocatalytic activity. For gas-surface interactions, available results suggest that using ferroelectrics as supports for catalytically active transition metals and oxides is another way to enhance catalytic activity. Finally, the possibility of incorporating ferroelectric switching into the catalytic cycle itself is described. In this scenario, a dynamic collaboration of two polarization states can be used to drive reactions that have been historically challenging to achieve on surfaces with fixed chemical properties (e.g., direct $\mathrm{NO}_{\mathrm{x}}$ decomposition and the selective partial oxidation of methane). These predictions show that dynamic modulation of the polarization can help overcome some of the fundamental limitations on catalytic activity imposed by the Sabatier principle.
\end{abstract}

Keywords: Ferroelectrics, Catalysis, Photocatalysis, Switchable surface chemistry, Surface reconstructions, Novel scaling relations 


\section{Introduction}

A polar surface arises when the electrical dipole associated with the bulk unit cell of a polar crystalline material has a non-zero component perpendicular to the surface i.e., $\vec{P}$. $\hat{n} \neq 0$, where $\vec{P}$ is the bulk polarization vector and $\hat{n}$ the surface normal. The emergence of bulk polarization is a consequence of the offset between the centers of the positive and negative charges in each unit cell [1, 2]. If this polarization were uncompensated, a constant electric field would develop across the material with an energy cost scaling with the thickness of the material and rapidly becoming unphysically large for thick samples. The solution is to accumulate equal amounts of compensating opposite charges $(\sigma= \pm \vec{P} . \hat{n})$ on the opposing polar surfaces to cancel the interior electric field so that the energy becomes independent of thickness. This can most simply be achieved through an electronic reconstruction: electrons or holes are transferred to the surfaces to compensate the polarization charge (Fig. 1) $[3,4,5,6,7,8,9,10]$. Over timescales where atoms move and can migrate to or away from the surfaces, an additional avenue for compensation becomes possible: atomic reconstruction. Here, atoms are adsorbed or removed from the surfaces. These adsorbates or vacancies make available additional electronic states for electrons or holes that more favorably accommodate them compared to the energy bands of the stoichiometric surfaces. For pristine surfaces under UHV, the adsorbates are typically species found in the bulk, for example oxygen anion adsorption on the positive surfaces of polar oxides, while under ambient conditions a range of foreign species must also be considered. Such defects then accept the electrons or holes and lower the total energy of the surface, becoming ions in the process (in some cases charged species may adsorb directly). Thus, the net effect of atomic reconstruction is to add ions to the surface that balance the polarization with a surface charge density of opposite sign and cancel the electric field inside the material. It is worthwhile to note that there are scenarios where the polarization may not be entirely compensated. For example, ultra-high vacuum (UHV) can limit atomic reconstructions through adsorption while optical and thermal excitations in large bandgap ferroelectrics can be insufficient to fully compensate the polarization through electronic reconstruction [11, 12, 13]. Another mechanism by which ferroelectric surfaces may avoid reconstruction is through the formation of nanodomains of alternate polarization which can eliminate the net polarization [14].

Polar surfaces almost always minimize their energy through some variety of atomic reconstructions rather than through purely electronic reconstructions $[3,15,16,17,18,19,20,21,22,23,24,25,26,27,28,29,30]$. These atomic reconstructions change the stoichiometry relative to the bulk termination which, in principle, can change the surface chemistry. Also, while the compensating charges eliminate the thickness-dependent component of the dipole, surface dipoles remain which can affect adsorption of polar molecules [31]. Ferroelectrics are a class of materials that have a spontaneous electric polarization vector (an order parameter) whose direction can be switched using an electric field and whose magnitude is temperature-controlled through the pyroelectric effect 
$[32,33]$. The modifications of ferroelectric surfaces due to the compensation of the surface charge density via adatoms, vacancies, surface dipoles and changes in surface electron density can all, in principle, alter the surface chemistry. Hence, ferroelectrics are promising candidates to achieve switchable and controllable surface physics and chemistry in which certain reactions can be turned on, off, or tuned.
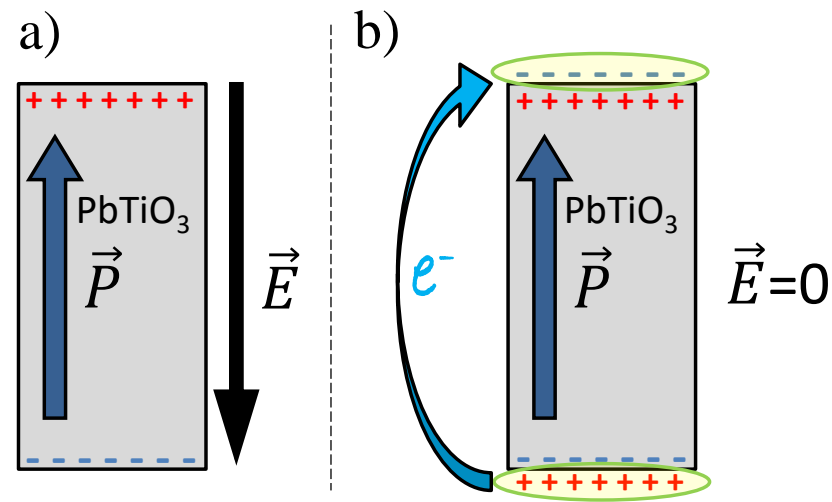

Figure 1: Schematic illustration of two scenarios for a ferroelectric surface. a) Unphysical scenario: a large depolarizing electric field exists across the entire material created by the uncompensated surface charges stemming from ionic displacements along the polarization direction. b) electronic reconstruction by electrons or holes for positive or negative polarization $\vec{P}$ which results in zero net electric field inside the material.

Since the mid $20^{\text {th }}$ century, the intriguing possibility of dynamically controlling surface chemistry and enhancing catalytic properties has turned ferroelectric and polar materials into the subject of numerous studies. Starting in 1952, Parravano observed anomalies in CO oxidation rates over ferroelectric sodium and potassium niobates near the Curie temperature of these materials (the second order transition temperature when the polarization vanishes) [34]. Next, in the 1960s, Stadler demonstrated that ferroelectric polarization can change the physical properties of supported metal films [35, 36]. The subject received renewed attention in the 1980s when Inoue et al. studied the effect of the substrate polarization on catalytic activity of supported metals and found higher $\mathrm{CO}$ oxidation rates for negatively poled surfaces, which they attributed to weaker $\mathrm{CO}$ adsorption for metal supported on the positively poled surface $[37,38]$. In the same timeframe, dramatic effects of the polarization direction on adsorption on polar, but not ferroelectric, materials were demonstrated [39, 40]. Using Temperature Programmed Desorption (TPD) measurements to study the adsorption of oxygen-containing molecules that possess strong dipole moments, such as formaldehyde and formic acid, on polar $\mathrm{ZnO}$ surfaces, Akhter et al. showed strong interactions with the Zn-polar (0001) face, a weaker interaction with a non-polar face, and only a weak interaction with the O-polar $(000 \overline{1})$ 
face [39]. Vohs et al. also studied $\mathrm{ZnO}$ polar surfaces using TPD and X-ray Photoelectron Spectroscopy (XPS) and showed that the Zn-polar crystal face readily oxidized both formic acid and formaldehyde while the O-polar face was unreactive [40].

The photocatalytic properties of ferroelectrics and polar materials have also attracted significant attention in the scientific community $[41,42,43,44,45$, $46,47,48,49,50,51,52,53,54,55,56,57,58,59,60]$. As far back as 1986, Inoue et al. studied photoassisted water decomposition on lead zirconate titanate (PZT) ceramics. In these experiments, 10-40 times higher $\mathrm{H}_{2}$ production rates were observed for positive relative to negative polarization [42]. The main underlying mechanism was determined to be the polarization-dependent band bending close to the ferroelectric surface that facilitates the separation of the electron-hole pair and, depending on the polarization direction, directs either electrons or holes to the surface. Thus, in positive polarization, enhanced electron transfer to the surface promotes the hydrogen evolution reaction. A photocatalyst with a semiconductor/metal/ferroelectric structure was studied in which the semiconductor $\left(\mathrm{TiO}_{2}\right)$ exhibits a higher efficiency for hydrogen formation for positive polarization in contrast to negative polarization [43]. In succeeding work on the ferroelectric semiconductor lead strontium zirconate titanate $\left(\mathrm{Pb}_{0.95} \mathrm{Sr}_{0.05} \mathrm{Zr}_{0.53} \mathrm{Ti}_{0.47} \mathrm{O}_{3}\right)$, the difference in hydrogen evolution activity on the positive compared to the negative surface was increased by more than two orders of magnitude [44]. In 2001, Giocondi et al. studied photochemical oxidation and reduction reactions on the surface of ferroelectric $\mathrm{BaTiO}_{3}$. For UV irradiation under aqueous solutions containing dissolved $\mathrm{Pb}^{2+}$ or $\mathrm{Ag}^{+}$cations, they observed accumulation of the oxidation product $\mathrm{PbO}_{2}$ and the reduction product $\mathrm{Ag}$ on opposite polarization domains in patterns that reproduced the underlying ferroelectric domain structure. This demonstrated the ability of the ferroelectric to preferentially direct photogenerated electrons or holes to the surface depending on the polarization direction [61,62].

Building on the results of Giocondi et al., the novel method of ferroelectric lithography was proposed in which ferroelectrics are utilized to fabricate multicomponent nanostructures (oxide substrates, metal nano-particles and organic molecules) $[63,64,65,66,67,68,69]$. In this method, ferroelectric polarization in the substrate is manipulated to control local electronic structure and chemical activity and is patterned with electron beams or probe tips. By using photo reduction from aqueous solution, metal nanoparticles are deposited on predefined locations (see Fig. 2). Organic molecules can then react selectively on these nano-particles. This process can in principle be repeated to develop even more complex structures.

All ferroelectrics are necessarily pyroelectric so that their polarization magnitude is temperature dependent [70,71]. Hence, heating or cooling a ferroelectric changes the balance of the surface compensating ions and surface polarization charges; as a result, the surface can temporarily find itself with extra charges for some time before it relaxes due to molecular adsorption/desorption or cancellation by the material's free carriers. As explained by Yun et al. [31], this leads to a sizable change in the surface dipole moment that can change surface 

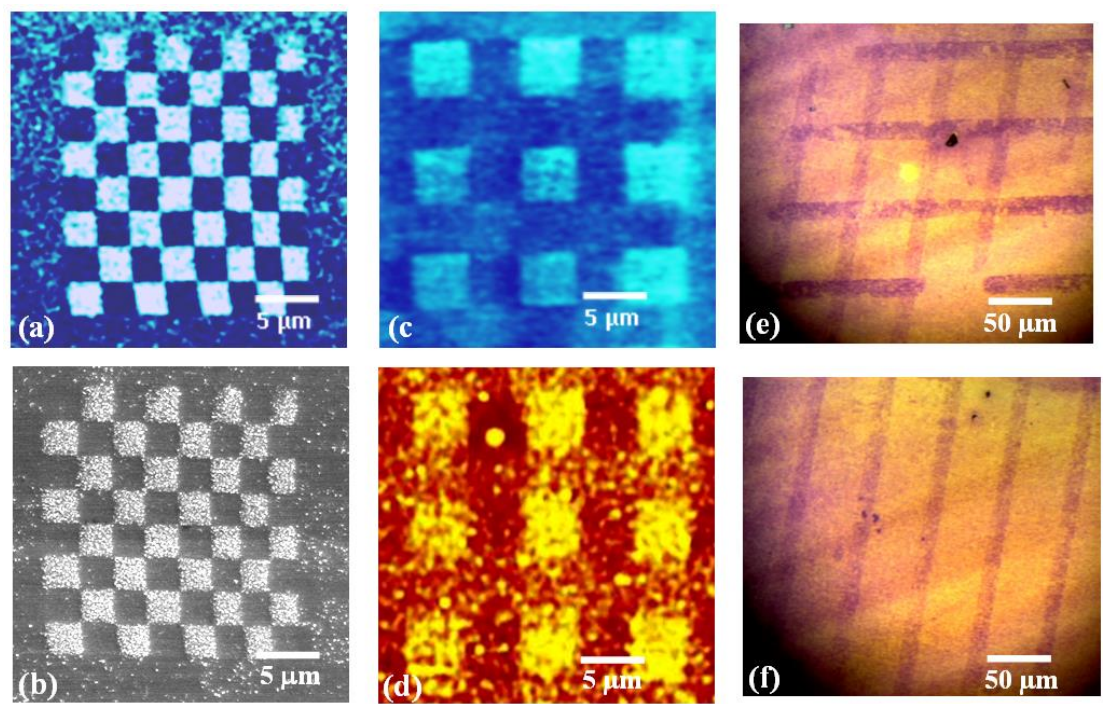

Figure 2: The domain orientation on PZT films visualized by scanning probe microscopy (a) and scanning electron microscopy (c) is correlated with the subsequent Ag photodeposition (b, d). Different microcontact stamps and subsequent photodeposition make grid like (e) and stripe like (f) silver patterns. Reprinted with permission from S. V. Kalinin, D. A. Bonnell, T. Alvarez, X. Lei, Z. Hu, R. Shao, and J. H. Ferris Advanced Materials, 16(9-10), 795-799.

chemistry. This effect is most noticeable in ferroelectrics with lower free carrier densities which strongly prefer external screening by adatoms (vacancies). This phenomenon has been exploited to create self-focusing, spatially stable, high energy $(170 \mathrm{keV})$ electron beams using $\mathrm{LiNbO}_{3}$ crystals in dilute gases [72], and to achieve "tabletop" nuclear fusion $[73,74,75]$. The basis of these experiments is the creation of a strong electric field near the ferroelectric surface which results from the temporary uncompensated charges on the surface of the ferroelectric created by heating or cooling [76, 77, 78, 79, 80, 81, 82, 83].

The seminal works briefly outlined above have motivated many fundamental studies on ferroelectrics and ferroelectric-supported thin films in recent years. In addition to many experimental studies, the development of better computational facilities, algorithms, software packages [84] and ab initio electronic structure methods, especially Density Functional Theory (DFT) and its derivatives, [85, $86,87,88,89,90]$, has led to a considerable number of theoretical studies in this field. Below, we review prominent recent experimental and theoretical results in the field of ferroelectric surface chemistry and provide an outlook on avenues that may potentially further advance this field. In particular, a strong potential benefit of ferroelectrics for catalysis can be their ability to deliver surfaces with dynamically switchable and tunable chemical properties. These systems may enable the realization of efficient processes for reactions such as $\mathrm{NO}_{x}$ direct decomposition or selective methane partial oxidation that have 
been challenging for conventional catalysts operating within the restrictions of the Sabatier principle which governs catalysis by surfaces with static chemical interactions.

\section{Bare Ferroelectric Surfaces}

\subsection{Surface Structure}

As noted above, most polar surfaces experience atomic and electronic reconstructions that depend on the polarization magnitude and direction. Furthermore, as the band gap increases, atomic reconstructions become more favorable than electronic reconstructions [91]. Since the common ferroelectric materials all have bandgaps in excess of $3 \mathrm{eV}[92,93,94,95,96]$, the expectation is that atomic reconstructions will dominate the charge compensation mechanism of undoped bulk materials at equilibrium. Within this context, Garrity et al. studied both electronically and atomically reconstructed $\mathrm{PbTiO}_{3}$ surfaces using DFT [3]. They found that the positively polarized $\mathrm{PbTiO}_{3}$ surfaces prefer to have either $0.5 \mathrm{ML} \mathrm{O}$ adatoms or $0.5 \mathrm{ML} \mathrm{Pb}$ vacancies, both electronegative defects, depending on the oxygen chemical potential; negatively polarized surfaces favor 0.5 ML electropositive oxygen vacancies. These atomically reconstructed, non-stoichiometric polar surfaces are in contrast to the non-polar paraelectric surface which prefers a stoichiometric state. Experimentally, a $(4 \times 1)$ reconstruction has been reported on the negative surface which is likely associated with arrays of oxygen vacancies on the surface [97]. Similar theoretical results have been reported by $\mathrm{Mi}$ et al. for $\mathrm{BaTiO}_{3}$ surfaces [98]. They have shown that the presence of oxygen vacancies at the $\mathrm{BaTiO}_{3}(001)$ surface preferentially stabilizes an inward pointing (negative) polarization. They used mirror electron microscopy (MEM) measurements [99], a variation of low energy electron microscopy (LEEM) with very low energy electrons that provides information on surface topography and work function, to confirm their theoretical results. We note that the surface structure of $\mathrm{BaTiO}_{3}$ is complicated by a myriad of observed reconstructions that closely mirror those seen on paraelectric $\mathrm{SrTiO}_{3}$; thus, many of the reconstructions are not primarily driven by charge compensation requirements $[100,101]$. Nonetheless, Chen et al. have recently used an evolutionary algorithm approach to computationally study a large number of reconstructed surfaces and have found a significant polarization dependence in the phase diagram [102] of ferroelectric $\mathrm{BaTiO}_{3}[103]$.

In 2008, Levchenko et al. used first-principle DFT calculations to study the surface reconstructions of ferroelectric $\mathrm{LiNbO}_{3}$ (0001) as a function of polarization [19]. Their surface phase diagrams predicted that the positive surface is $-\mathrm{Nb}-\mathrm{O}_{3}-\mathrm{Li}_{2}$ terminated ( $\mathrm{Li}$ on top) and the negative surface is $-\mathrm{Li}-\mathrm{O}$ terminated (see Fig. 3). Their theoretical model, which indicates an excess of $\mathrm{Li}$ and $\mathrm{O}$ on the positive surface relative to the negative one, explains the experimental observations of Lushkin et al. [104] who discovered a difference in relative evaporation rates of $\mathrm{LiO}, \mathrm{Li}$ and $\mathrm{O}_{2}$ from $\mathrm{LiNbO}_{3}$ surfaces depending on the polarization direction. This theoretical model is also consistent with 
low energy ion scattering measurements that puzzlingly indicated that both the positive and negative surfaces were terminated by oxygen [105, 106]. Because low energy ion scattering is insensitive to $\mathrm{Li}$, such ion scattering spectra reveal the surface $\mathrm{O}$ atoms with little evidence of $\mathrm{Nb}$ independent of the polarization. Separately, by using frequency-modulation atomic force microscopy (FM-AFM) $[107,108]$ to achieve atomic resolution on $\mathrm{LiNbO}_{3}$ in an aqueous environment [109] in combination with DFT calculations, Sanna et al. showed that high temperature annealing drives off external adsorbates and unmasks the intrinsic surface atomic reconstructions that lower the surface charge and energy [110]. Specifically, they observed a $(\sqrt{7} \times \sqrt{7}) R 19.1^{\circ}$ reconstruction for the $(000 \overline{1})$ surface that they assigned to a superstructure of added Li-O groups on the surface.
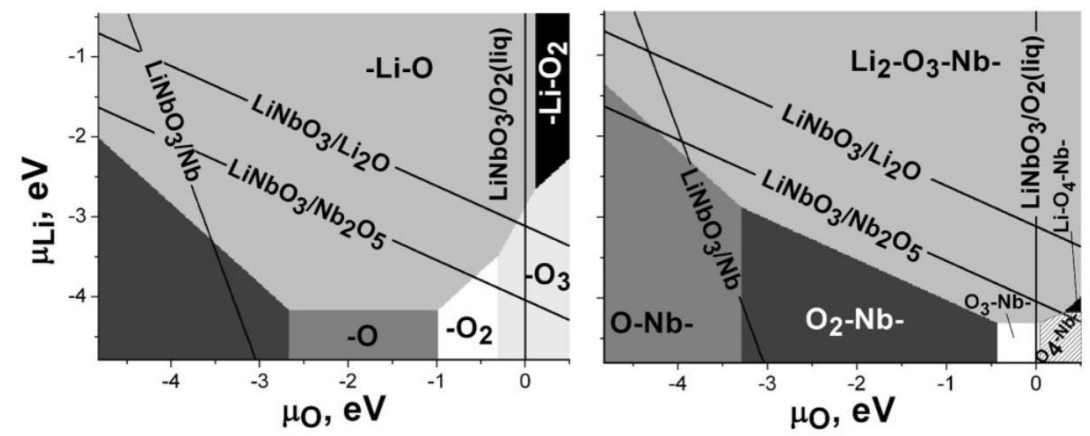

Figure 3: Phase diagrams showing the thermodynamically stable surface terminations of negative (left) and positive (right) surfaces of $\mathrm{LiNbO}_{3}(\mathrm{LN})(0001)$ as a function of $\mathrm{Li}$ and O chemical potentials. The terminations are labeled with hyphens. The unlabeled region on the left corresponds to a Nb-terminated surface. The black lines denote boundaries beyond which new bulk phases (shown on corresponding lines) start to precipitate on the surfaces. The physically important region where bulk LN is stable is within the trapezoid formed by the black lines. Reprinted with permission from S. V. Levchenko, and A. M. Rappe, Physical review letters, $100(25), 256101$.

As explained above, both experiment and theory reveal a rich array of surface structures that depend on the ferroelectric polarization direction as well as the surrounding environment. Although the surface structures can be complex, a unifying theme is that in the absence of extrinsic compensating charges the surface stoichiometry adjusts to compensate the bulk polarization: oppositely poled surfaces are compositionally and structurally distinct $[111,112,19]$. Therefore, in addition to the electronic changes induced by polarization (induced free carriers and band-bending), structural and compositional differences can lead to a polarization-dependent surface chemistry on ferroelectric (polar) surfaces [113, 3, 114, 115, 29, 116, 117, 118, 119, 120, 121, 122].

\subsection{Polarization dependent adsorption and desorption}

In 2007, Yun et al. compared the adsorption of polar and non-polar molecules on oppositely poled, atomically reconstructed $\mathrm{LiNbO}_{3}$ surfaces $[123,31]$. The 
results revealed that the interaction of polar molecules with the surface is dominated by electrostatic interactions with the surface dipole. As illustrated in the TPD results in Fig. 4, polar acetic acid desorption peaked roughly $100 \mathrm{~K}$ higher on the positive surface while non-polar dodecane desorption was independent of the polarization direction. The $100 \mathrm{~K}$ higher desorption peak temperature for acetic acid on the positive surface generally suggests substantially stronger adsorption on this surface. To quantify this difference, the desorption peak temperatures, $T_{P}$, were measured as a function of heating rate $\beta$. The data for dodecane were nearly identical for the two surfaces and as anticipated: the desorption activation energy was $0.80 \mathrm{eV}$ per molecule and the pre-exponential $10^{15} \mathrm{~s}^{-1}$, both values typical of large alkanes. Meanwhile, the data for the polar molecules acetic acid and 2-propanol were consistent with stronger adsorption on the positively poled surface (steeper slope) but also suggested desorption pre-exponentials at least 10 orders of magnitude lower than typically expected. When this is taken into account, the difference in desorption activation energies between the positive and negative surfaces becomes a modest $0.08 \mathrm{eV}$ per molecule for acetic acid.

The anomalously low apparent desorption pre-exponential could be explained by a temperature dependent heat of adsorption. Because ferroelectric materials are also pyroelectric, large fields can develop at the surfaces of these materials when they are heated or cooled in vacuum as described in the Introduction. If no relaxation takes place, the net dipole would increase by roughly $285 \mathrm{e} \AA / \mathrm{K}$ during heating. Such a large change would alter how strongly polar molecules adsorb as the sample is heated but would have a negligible effect on a non-polar, weakly polarizable molecule such as dodecane. Subsequent work function measurements on $10 \mathrm{~nm}$ thick ferroelectric and pyroelectric PZT films on a metallic substrate supported this picture [124]. In these experiments, the work function reversibly increased by $\approx 300 \mathrm{meV}$ when a positively poled film was heated from 300 to $400 \mathrm{~K}$, consistent with the model that heating decreases the bulk polarization and leaves excess compensating charges on the surface that induce an inward pointing dipole across the PZT and increase the work function of the metal (See Fig. 5). It is easy to see how scaling these effects to the surfaces of bulk crystals can lead to enormous surface potentials that affect how strongly polar molecules interact with the surface. Taking this into account, the results indicate that the chemical interactions with the atomically reconstructed surfaces are weak and that intrinsic differences between the heats of adsorption on the oppositely poled surfaces exist but are not large.

Zhang et al. showed that differences between the behavior of oppositely poled surfaces cannot be solely due to electrostatic interactions [125, 126]. Using a combination of infrared microscopy and spatially resolved x-ray absorption near edge spectroscopy, in 2010 they studied the polarization-dependent adsorption of polar d-cysteine molecules on periodically poled $\mathrm{LiNbO}_{3}$ during isothermal deposition from an aqueous $1 \mathrm{M}$ solution and observed preferential dcysteine deposition on positive domains [126]. Since the deposition was isothermal and without illumination, it was concluded that the polarization dependent interaction between d-cysteine and the ferroelectric surface could not be 

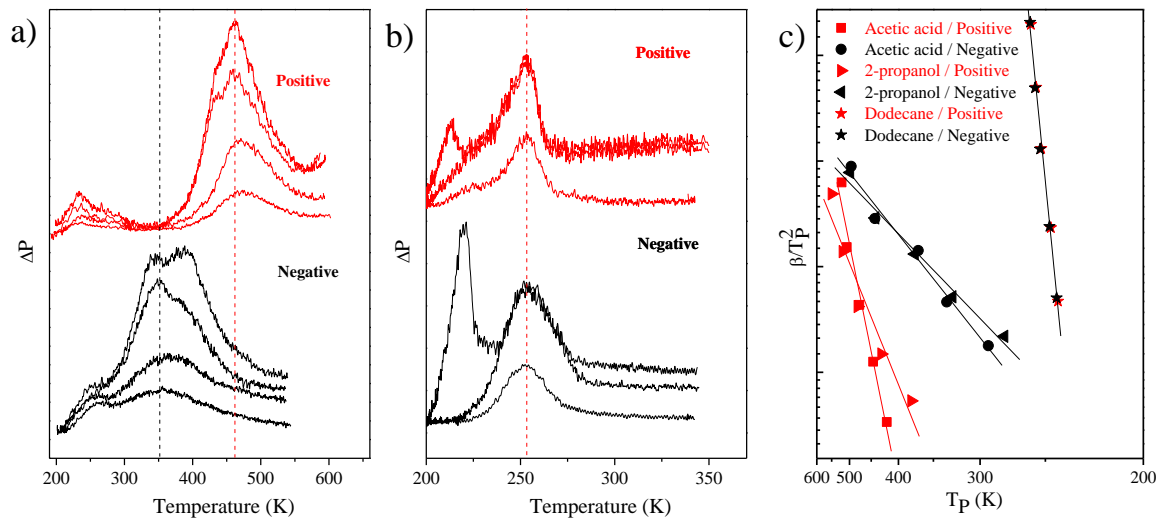

Figure 4: a) Comparison of acetic acid TPD curves for positively (top, red) and negatively (bottom, black) poled $\mathrm{LiNbO}_{3}$ (0001). The sequence of curves illustrate the coverage dependence on the two surfaces; the vertical dashed lines highlight the difference in desorption peak temperatures. b) Dodecane TPD curves for several different coverages for positively (top, red) and negatively (bottom, black) poled $\mathrm{LiNbO}_{3}$. The low temperature peak is due to multilayer desorption. The vertical dashed line shows that monolayer desorption has a peak at the same temperature on both surfaces, so that differences in the multilayer peak temperatures are due to the higher coverages on the negative surface. c) Redhead plots for acetic acid, 2-propanol, and dodecane on positively and negatively poled $\mathrm{LiNbO}_{3}(0001)$ where $\beta$ is the heating rate and $\mathrm{T}_{P}$ the desorption peak temperature. Adapted from Y. Yun and E.I. Altman, J. Am. Chem. Soc. 129 (2007) 15684.

due to the pyroelectric effect or photo-induced charges. They attributed this polarization dependent interaction to a dipole-dipole interaction between the surface and the molecule as well as to changes in surface chemistry related to polarization-dependent variations in $\mathrm{Li} / \mathrm{Nb}$ concentrations. The latter conclusion was reinforced by their 2011 work in which they studied the chemisorption of two isomers of diiodobenzene on ferroelectric polymer surfaces where one of the isomers (1,2-diiodobenzene) was polar and the other (1,4- diiodobenzene) had zero net dipole [125]. They showed that the non-polar isomer preferentially adsorbed on positive domains at $150 \mathrm{~K}$ while the polar isomer strongly preferred the negative polarization. As a result of the polarization specific interaction of the non-polar isomer with the positive surface, they concluded that surface chemistry and not simply the surface dipoles (i.e., simple electrostatics) matters in these chemisorption processes. These results from Zhang et al. and Yun et al. on the interactions of non-polar molecules with the polar ferroelectric surface might initially appear contradictory. However, Yun et al. intentionally choose chemically inert dodecane as their non-polar molecule to isolate electrostatic contributions while the non-polar molecule chosen by Zhang et al., (1,4diiodobenzene), is more reactive and hence its adsorption would be expected to respond more strongly to changes in surface structure and stoichiometry.

In 2009, Garra et al. studied the interaction of both $\mathrm{H}_{2} \mathrm{O}$ and $\mathrm{CH}_{3} \mathrm{OH}$ with 


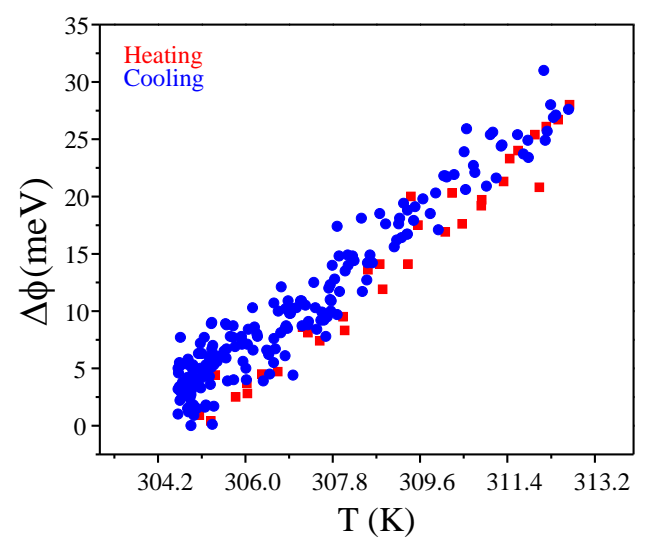

Figure 5: Plot of the change in work function of a $\mathrm{SrRuO}_{3}$ (001) surface covered by $10 \mathrm{~nm}$ of positively poled PZT as the temperature was increased and decreased. Color online. Reprinted with permission from M.W. Herdiech, H. Monig and E.I. Altman, Surf. Sci. 626 (2014) 53.

$\mathrm{LiNbO}_{3}(0001)$ surfaces and discovered that this interaction was both coverage and polarization dependent [127]. The polarization dependence of the zero coverage desorption energies was estimated to be $\approx 0.05 \mathrm{eV}$ per molecule higher on the positively poled surface. Three years later, Sanna et al. used ab initio DFT calculations to study water adsorption on $\mathrm{LiNbO}_{3}$ (0001) surfaces and found that adsorption energies, binding configurations and molecular mobility change as a function of polarization [128]. Their results qualitatively agreed with the TPD measurments by Garra et al., with higher water adsorption energies on the positively poled surface, $1.28 \mathrm{eV}$ per molecule on the positive versus $0.61 \mathrm{eV}$ per molecule on the negatively poled surface. They mentioned that although their first principle calculations agreed qualitatively with the experiment, the computed polarization dependence was an order of magnitude larger than what was observed experimentally. In fact, this discrepancy is a general trend when one compares computational and experimental results on ferroelectrics, a fact that we will revisit below. In other work, Zhao et al. used the adsorption of 2-fluoroethanol on $\mathrm{BaTiO}_{3}$ to assess the effect of polarization on reaction pathways [129]. The fluorinated ethanol adsorbs as an alkoxide that can react upon heating to produce acetaldehyde, ethylene, and adsorbed fluorine atoms which remain on the surface. Using TPD measurments, they observed a polarization dependent activation energy for acetaldehyde production which was $\sim 0.04 \mathrm{eV}$ per molecule higher on the positively poled surface.

The preceding works focused on adsorption energies and desorption rates. In 2008, Vohs et al. showed that polarization can also affect adsorption rates [130]. Specifically, they observed domain dependent sticking coefficients for $\mathrm{CH}_{3} \mathrm{OH}$ and $\mathrm{CO}_{2}$ on $\mathrm{BaTiO}_{3}$ and $\mathrm{Pb}\left(\mathrm{Ti}_{0.52} \mathrm{Zr}_{0.48}\right) \mathrm{O}_{3}$ surfaces using TPD and scanning surface potential microscopy, supported by first-principles calculations. 
Also in 2008, Zhao et al. used TPD measurements to observe a polarization dependent sticking coefficient for ethanol on ferroelectric $\mathrm{BaTiO}_{3}$ which was higher on positively poled surfaces [131]. The TPD peak shapes and the relative product yields were also polarization dependent suggesting that ferroelectric polarization may affect the intrinsic reactivity of the surface rather than simply changing the number of active sites. Recently, Stoflea et al. performed XPS and piezoresponse force microscopy (PFM) on PZT (001) single crystals and found that contaminants such as fatty acids, alcohols, and esters with oxygen containing anionic heads adsorbed preferentially on positive domains rather than negative ones [132].

As noted above, although the polarization direction can change the surface chemistry and modify adsorption and desorption for both polar and non-polar molecules [133], the magnitude of the effect in experiments is rather small and

at most a few tenths of an eV. In parallel, the polarization dependences of binding energies computed by DFT are almost always overestimated in comparison [134, 128]. This difference is likely explained by the work by Garrity et al. who showed that both non-stoichiometric (atomically reconstructed) and stoichiometric (purely electronically reconstructed) surfaces show polarization dependent $\mathrm{CO}_{2}$ and $\mathrm{H}_{2} \mathrm{O}$ adsorption, but that the effect is much stronger on surfaces that have only undergone electronic reconstruction by free carriers [3]. In other words, the purely electronically reconstructed surfaces are not as stable as the atomically reconstructed ones and are thus more chemically active and more chemically differentiated. We offer the simple conclusion that the overestimation of polarization dependence of adsorption energies is probably due to differences in the surfaces that are computationally modeled versus those that are stable under the conditions that the experiments are carried out.

\subsection{Photocatalysis}

As mentioned above, the photocatalytic properties of ferroelectrics have attracted much attention in the scientific community since the 1980s [135, 136, $137,138,139,140]$. In 2011, Schultz et al. studied the photochemical reduction of $\mathrm{Ag}$ from an aqueous solution on $\mathrm{BiFeO}_{3}$ as a function of crystal and ferroelectric domain orientation. They observed that silver is preferentially reduced on domains with positive polarization. They also showed that the amount of reduced silver depends on whether the component of the polarization normal to the surface is positive or negative but is relatively insensitive to the crystal orientation [141]. In a study of photochemical reduction of $\mathrm{Ag}$ by $\mathrm{Ba}_{1-x} \mathrm{Sr}_{x} \mathrm{TiO}_{3}$ $(x=0-1)$, Bhardwaj et al. discovered that $\mathrm{BaTiO}_{3}(x=0)$ selectively reduced $\mathrm{Ag}$ on positive domains. By continuously increasing $x$ (which decreases the magnitude of the bulk polarization), they found a continuous change in reduction pattern from spatially selective to uniform [142]. They also discovered a local maximum in reactivity at $x=0.26$, near the cubic-tetragonal phase boundary: this corresponds to an anomalously high dielectric constant which results in a larger space-charge region [60, 143] and thus an increased reactivity. As mentioned above, one of the main mechanisms behind the enhanced photochemical reactivity of ferroelectric surfaces is the special (polarization dependent) band 
bending that happens near the ferroelectric surface as a result of the internal electric fields associated with spontaneous polarization. In a comprehensive review of the subject by $\mathrm{Li}$ et al., four sources for this internal field have been presented: a) ferroelectric polarization, b) polar surfaces, c) p-n junctions [144, 145] and d) polymorph junctions [146]. As can be seen in Fig. 6, as a result of the band bending caused by these fields, the transfer of either holes or electrons to the surface can be facilitated. Thus, ferroelectric surfaces can exhibit spatially selective reducing or oxidizing photochemical activity.

(a)

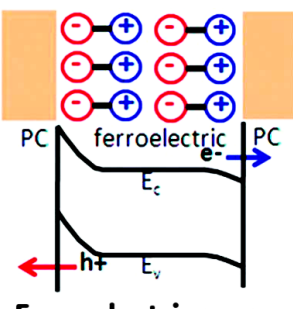

Ferroelectric core

(c)

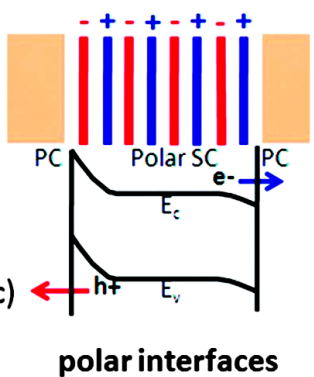

(b)

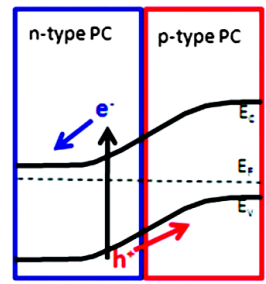

p-n junction

(d)

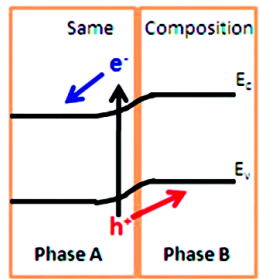

polymorph junctions

Figure 6: Photogenerated charge carrier separation can be enhanced by internal fields: (a) ferroelectric polarization; (b) p-n junctions; (c) polar surfaces; and (d) polymorph junctions. (PC: photocatalytic active materials; SC: semiconductor). Reprinted with permission from L. Li, P. A. Salvador, and G. S. Rohrer, Nanoscale, 6(1), 24-42.

A recent study by Park et al. on a polarized particulate suspension (PS) system shows that the photocatalytic activity of $\mathrm{K}_{0.5} \mathrm{Na}_{0.5} \mathrm{NbO}_{3}$ (NKN) for $\mathrm{H}_{2}$ evolution improves by a significant factor of 7.4 due to polarization (relative to the paraelectric phase) [147]. They discover that the positively poled surface plays an essential role in promoting the photocatalytic activity. They attribute this to the improvement in the charge-separation caused by the photovoltaic effect and the efficient charge transfer characteristics promoted by band bending. The physical picture they propose begins with NKN being a n-type material so that one expects upward band bending (Schottky barrier formation) at the water-ferroelectric interface; however, the internal electric fields due to polar charge compensation near the surface induce downward band bending on the positive surface, so that the partial cancellation of the barrier facilitates electron transfer to the electrolyte and thus enhances the hydrogen evolution reaction (HER). Another benefit of ferroelectrics for photocatalysis, also pointed 
out by Park et al., is the anomalously high photo voltages (APV) that can be achieved by these materials: the voltage output along the polarization direction can be vastly larger than the band gap of the ferroelectric material [148]. These properties have also raised interest in ferroelectrics for photovoltaic devices $[149,150,151,152,153,154,155,156,157]$, where it has been shown that the switchable nature of ferroelectric polarization can lead to switchable photovoltaic properties [158, 159, 160, 161, 162, 163].

There has also been a number of studies that describe enhanced photocatalytic activity at domain and grain boundaries in ferroelectrics. In 2007, Jones et al. investigated the photo-reduction of silver salts on highly heterogeneous lead zirconate titanate (PZT) [164]. They showed that the greatest deposition rates occurred at grain boundaries, likely due to a combination of band bending and a positive electric field present at the grain boundaries that causes the electrons to migrate towards them [164, 165]. In 2009, Haussmann et al. studied single crystal $\mathrm{LiNbO}_{3}$ and showed that photochemical reduction of elemental metals from ionic solutions on these surfaces was confined to ferroelectric $180^{\circ}$ domain walls [166]. Sun et al. studied periodically poled lithium niobate (PPLN) and showed that for all above-gap-excitation wavelengths, deposition occurs on surfaces of either polarity as well as at the domain boundaries, but that the deposition density is greatest at the domain boundaries [11]. Sun et al. attribute the differences between the PPLN results and those for PZT and barium titanate (in which Ag deposition occurs predominantly on positive polarization $[167,142,168])$ to different polarization screening mechanisms. For materials such as PZT, the concentration of defects including oxygen vacancies is estimated to be relatively high so that the screening/compensation of the polar surface charge is done inside the material and leads to a sizable band bending. However, for materials such as lithium niobate which have a lower density of near surface defects, the band bending is weaker and the polarization charges are compensated primarily by external adsorbates. The external screening and weaker band bending of single crystal lithium niobate introduces an enhanced electric field at the domain boundary which leads to migration of electrons to the domain boundary and consequently enhanced Ag deposition along the domain boundaries. Sun et al. also show that high doses of oxygen implantation increases the defect concentration and changes the screening mechanism from external to internal, after which the deposition pattern becomes more similar to photo-deposition of Ag on PZT [169].

\section{Ferroelectrics modified by transition metals}

Despite the success of bare ferroelectric materials in aqueous phase photocatalysis, gas phase heterogeneous catalysis using ferroelectrics has been less promising since the effect of the polarization on adsorption energies was found to be small. This is not surprising since although the polarization surface charge density $(\sigma=\vec{P} . \hat{n})$ is large in canonical ferroelectrics and is in the range of $0.5-1.0$ electrons per surface unit cell, over time the ferroelectric surface becomes passivated through atomic reconstructions and is rendered less reactive. Hence, even 
though first principles calculations on electronically reconstructed surfaces show pronounced polarization dependent behavior, such a surface phase is not easily accessible when the surface has time to relax to its thermodynamically preferred state. One way to avoid this problem is to try to combine the surface reactivity of transition metals with the polarization dependent properties of ferroelectrics. Here, one supports an ultra-thin transition metal film on a polar or ferroelectric substrate in order to modulate the transition metal surface chemistry through the substrate polarization. A specific example is the 1988 work by Didziulis et al. on $\mathrm{Cu} / \mathrm{ZnO}[170]$ which showed that although $\mathrm{CO}$ chemisorption on highly dispersed $\mathrm{Cu}$ on the $\mathrm{Zn}$-polar (0001) surface and the non-polar (1010) surface is very similar to $\mathrm{CO}$ chemisorption on bulk $\mathrm{Cu}$, it is much weaker for $\mathrm{Cu}$ on the O-polar $(000 \overline{1})$ surface. This approach has also been applied to aqueous phase photocatalysis [42]. This overall idea has provided a significant avenue of research into novel and potentially switchable surface chemistry and catalysis in the last few decades.

\subsection{Polarization dependent adsorption and desorption}

In 1990, Roberts et al. studied CO adsorption on Pt overlayers placed on different substrates: non-polar $\alpha-\mathrm{Al}_{2} \mathrm{O}_{3}$ (0001), positively polarized $\mathrm{ZnO}$ (0001), and negatively polarized $\mathrm{ZnO}(000 \overline{1})$ [171]. Using Auger electron spectroscopy (AES) and transmission electromicroscopy (TEM), they observed that Pt grows as a $2 \mathrm{D}$ film at $300 \mathrm{~K}$ on both polar surfaces in contrast to $\mathrm{Pt}$ on the non-polar sapphire surface on which it tends to form 3D particles. Using transmission electron diffraction (TED), they also showed that Pt grows as an ordered overlayer on $\mathrm{ZnO}$ crystals. Heating the $\mathrm{Pt} / \mathrm{ZnO}$ surfaces resulted in the formation of $3 \mathrm{D}$ Pt particles starting at $\sim 650 \mathrm{~K}$. The TPD curves for $\mathrm{CO}$ from high $\mathrm{Pt}$ coverages $(>1 \mathrm{ML})$ and from $\mathrm{Pt}$ particles formed by heating thinner films were shown to be identical to $\mathrm{CO}$ from $\mathrm{Pt}$ on the non-polar sapphire surface. For submonolayer coverages prior to Pt particle formation, however, the TPD curves for positively polarized $\mathrm{ZnO}$ (0001) shifted down by $\sim 60 \mathrm{~K}$ compared to Pt on negatively poled $\mathrm{ZnO}(000 \overline{1})$. They interpreted this shift as an indicator of a chemical interaction between $\mathrm{Pt}$ and the $\mathrm{Zn}^{2+}$ ions which are in direct contact on the (0001) surfaces. Subsequent work by Vohs and co-workers on Pd on $\mathrm{ZnO}(0001)$ and non-polar (1010) reinforced this conclusion [172, 173]. They found evidence of Pd-Zn alloy formation after heating above $700 \mathrm{~K}$ that was more pronounced on the $\mathrm{Zn}$-polar face which in turn had a larger effect on $\mathrm{CO}$ adsorption on the metal.

In 2007, Kolpak et al. used DFT to study polarization effects on the surface chemistry of $\mathrm{PbTiO}_{3}$-supported ultrathin $\mathrm{Pt}$ films [174]. They studied chemisorption of a variety of molecules and atoms and discovered that flipping the substrate polarization dramatically changed the computed chemisorption energies of $\mathrm{CO}, \mathrm{O}, \mathrm{C}$, and $\mathrm{N}$ and altered the reaction pathways for dissociation of $\mathrm{CO}, \mathrm{O}_{2}, \mathrm{~N}_{2}$, and NO. In particular, they suggested that Pt could be made to dissociate CO, a reaction that does not normally occur on Pt surfaces. They suggested that by exploiting the polarization effect, one can optimize the metal-molecule interaction to maximize activity (i.e., achieve the peak of the 
well known "volcano plot" of activity versus binding energy) to make a more efficient catalyst $[175,176,177,178,179]$. The main issue with this scheme is that to see a significant effect the Pt film must be ultrathin. The polarization effect is strongest for a monolayer ( $\sim 1 \mathrm{eV}$ binding energy shift), and it becomes almost negligible for films four or more layers thick ( $>1 \mathrm{~nm}$ thickness). Unfortunately, experimental endeavors to create such ultrathin transition metal films on ferroelectric oxides reveal a strong thermodynamic drive for the transition metals to form bulk-like crystalline nanoparticles. For such particles, the polarization effect essentially vanishes due to the weak coupling to the ferroelectric substrate. In 2009, Yun et al. studied the growth of ultrathin Pd layers on positively and negatively poled ferroelectric $\mathrm{LiNbO}_{3}$ (0001) surfaces [180]. Using low energy ion scattering, they discovered that the $\mathrm{Pd}$ tended to form threedimensional clusters on both positively and negatively poled substrates even at the lowest coverages. They also investigated the chemical properties of these Pd clusters using CO TPD, but found no sensitivity to the direction of substrate polarization. Lastly, they studied the electronic structure of the Pd clusters as a function of their size and observed no effect of the substrate polarization and a cluster size dependence that was characteristic of an inert support; e.g. $\alpha-\mathrm{Al}_{2} \mathrm{O}_{3}$.

In the same year, Zhao et al. studied the same system using a combination of Auger electron spectroscopy (AES) and TPD [181]. They found that at 300 $\mathrm{K}$, vapor-deposited $\mathrm{Pd}$ films grew in a layer-by-layer fashion on the negatively polarized $\mathrm{LiNbO}_{3}(0001)$ surface, in contrast to the positively poled surface where they formed 3D clusters for otherwise identical growth conditions. They attributed this difference to the different interaction energies between Pd and the positive and negatively poled surfaces due to differences in the composition of these surfaces. They also studied the thermal stability of these systems: even on the negatively poled surfaces, the Pd film started to agglomerate into 3D particles at $\sim 425 \mathrm{~K}$. In 2011, Kim et al. used DFT and kinetic Monte Carlo simulations to study the same $\mathrm{Pd}$ on $\mathrm{LiNbO}_{3}$ (0001) system [114]. For positive polarization, they found that $\mathrm{Pd}$ favored a clustered configuration, while for negative polarization $\mathrm{Pd}$ adsorbed in a more dispersed pattern due to suppression of diffusion and agglomeration. They traced back this disparity to the different adsorption geometries of $\mathrm{Pd}$ on $\mathrm{LiNbO}_{3}$ due to the differing atomic reconstructions of the positive and negative surfaces [19]. They explained that for the negative surface, a strong bond between $\mathrm{Pd}$ and extra surface $\mathrm{O}$ led to a larger energy barrier for diffusion and agglomeration. Because formation of large $\mathrm{Pd}$ clusters is thermodynamically favorable on $\mathrm{LiNbO}_{3}$ surfaces regardless of polarization state, they noted that it would be difficult to reversibly switch the Pd catalytic activity using ferroelectric polarization [114].

\subsection{Photocatalysis}

Several groups have sought to enhance the photocatalytic activity of ferroelectrics by adding transition metal catalysts. In 2013, Cui et al. studied the photocatalytic activity of $\mathrm{BaTiO}_{3}$ by measuring the decomposition rate of the 
typical dye molecule Rhodamine B under simulated solar light [182]. They observed that increasing the fraction of the material in the ferroelectric tetragonal phase through annealing tripled the photocatalytic catalytic activity. They also discovered that photochemical deposition of nanostructured Ag on the ferroelectric substrate significantly increases the photocatalytic activity of the material for organic decomposition reactions (see Fig. 7). Recently, Su et al. studied the novel photocatalytic properties of nanoscale ferroelectric $\mathrm{BaTiO}_{3}$ [183]. The nanoscale $\mathrm{BaTiO}_{3}$ has a large surface area which benefits catalysis, but the ferroelectric polarization of the nanoparticles is reduced compared to the bulk. By comparing behaviors above and below the Curie temperature, they showed that ferroelectricity could enhance the photocatalytic activity. They also discovered that coating $\mathrm{BaTiO}_{3}$ with $\mathrm{Ag}$ can enhance the photocatalytic activity even further $(>100 \%)$. They attributed this enhancement to the combined benefit of ferroelectricity, synergistic effects, local surface plasmon resonances, and specific charge-transfer kinetics in the $\mathrm{Ag}_{-} \mathrm{BaTiO}_{3}$ hybrid nanoparticles. There have also been a number of studies on decorating $\mathrm{BiFeO}_{3}$, one of the most promising multiferroic materials $[184,185]$ with a relatively narrow bandgap that may prove useful for visible-light-driven photocatalysis [186, 187, 188, 189], with Au and Ag nanoparticles $[190,191]$. A remarkably enhanced photocatalytic behavior, relative to the bare $\mathrm{BiFeO}_{3}$, was reported for both cases.

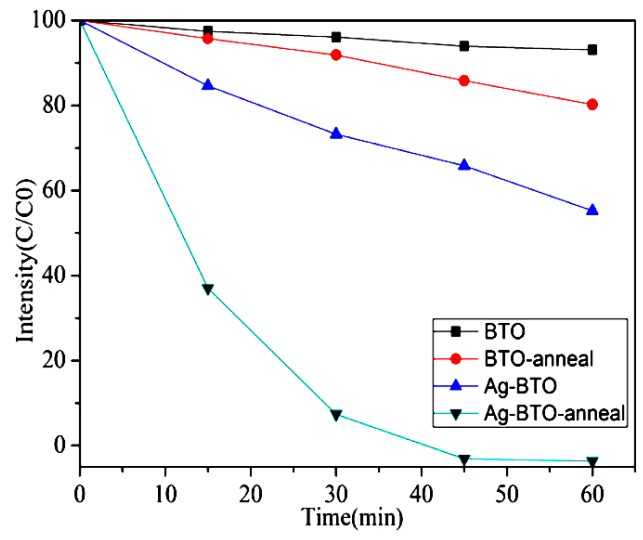

Figure 7: Photodecomposition profiles of the dye molecule Rhodamine B with different catalysts under illumination: dye concentration normalized to the initial concentration versus time. Catalysts containing more tetragonal ferroelectric $\mathrm{BaTiO}_{3}$ after annealing show higher activity, especially when modified by Ag nanoparticles. Reprinted with permission from Y. Cui, J. Briscoe, and S. Dunn, Chemistry of Materials, 25(21), 4215-4223.

\section{Oxides on Ferroelectrics}

As explained in the previous section, key difficulties with enhancing ferroelectric reactivity by adding transition metals are that (i) the maximum effect is achieved in monolayer films and rapidly tapers off with thickness, and (ii) the 
lack of wetting leads to the formation 3D transition metal nanoparticles on the ferroelectric surface; the nanoparticles, being primarily bulk like, couple only weakly to the ferroelectric. An alternate idea is to deposit chemically active metal oxide films on oxide ferroelectrics. Since they belong to the same class of materials and have lower surface tensions than transition metals, ultrathin oxide thin films can be grown more easily and with higher stability on oxide ferroelectric surfaces. In particular, recent advances in epitaxial growth methods, e.g., Molecular Beam Epitaxy (MBE) [192, 193, 194] and Atomic Layer Deposition (ALD) [195, 196, 197, 198], have made it possible to form structurally well-defined complex transition metal oxides of controlled thickness on ferroelectrics. In addition, metal oxides usually have longer screening lengths than transition metals which can enhance the coupling to the substrate's polarization and extend the impact of the ferroelectric to at least several atomic layers [199, 115].

In the last few decades, numerous experimental and theoretical studies have examined the effects of ferroelectric substrates on the physics and chemistry of thin oxide films. For example, Ahn et al. studied the ferroelectric field effect in epitaxial $\mathrm{SrCuO}_{2}$ and $\mathrm{SrRuO}_{3}$ thin films on PZT and found reversible and nonvolatile resistance changes approaching $10 \%$ for $30 \AA$ thick films when the ferroelectric polarization was reversed $[200,199]$. This modification was related to the change in the number of free carriers in the conduction bands of the thin films. In principle, such changes in the occupancy of bands near the Fermi level can also significantly affect surface chemistry and catalysis. In 2004, Zhang et al. demonstrated that the rates of oxidation and reduction reactions catalyzed by the surface of a $\mathrm{SnO}_{2}$ nanowire (configured as a field-effect transistors) could be varied by manipulating the electron density in the wire by applying a gate voltage [201, 202]. A much larger effect can be expected due to the surface charge induced by ferroelectric polarization, instead of a gate voltage [203, 204, 205], as Ahn et al. pointed out [194]: even a moderate ferroelectric polarization leads to a huge surface charge density of $\sim 1.5 \times 10^{14}$ electron $/ \mathrm{cm}^{2}$ and a concomitant large internal electric field of $\sim 300 \mathrm{MV} / \mathrm{cm}$. Further, the remnant polarization in a ferroelectric obviates the need for a gate voltage.

\subsection{Polarization dependent adsorption and desorption}

In 2013, Garrity et al. employed DFT calculations to study transition metal oxide monolayers of the form $\mathrm{BO}_{2}$ (e.g., $\mathrm{RuO}_{2}$ ) that can continue the $\mathrm{ABO}_{3}$ perovskite structure of ferroelectric $\mathrm{PbTiO}_{3}[3]$. They found that the surface chemistry of these monolayers couples strongly to the ferroelectric polarization of the substrate; for example, the binding energy of oxygen decreases by $1 \mathrm{eV}$ per atom when one flips the polarization direction from positive to negative. They proposed that this reversible chemistry can be used to capture $\mathrm{CO}_{2}$ molecules in one polarization state and then release them in the opposite polarization state (i.e., as part of a $\mathrm{CO}_{2}$ sequestration system [206, 207]). As described above, the polarization dependence of binding energies is much more significant on surfaces with purely electronic reconstruction when compared to those passivated by atomic reconstructions [3]. Recently, this theoretical work was extended 

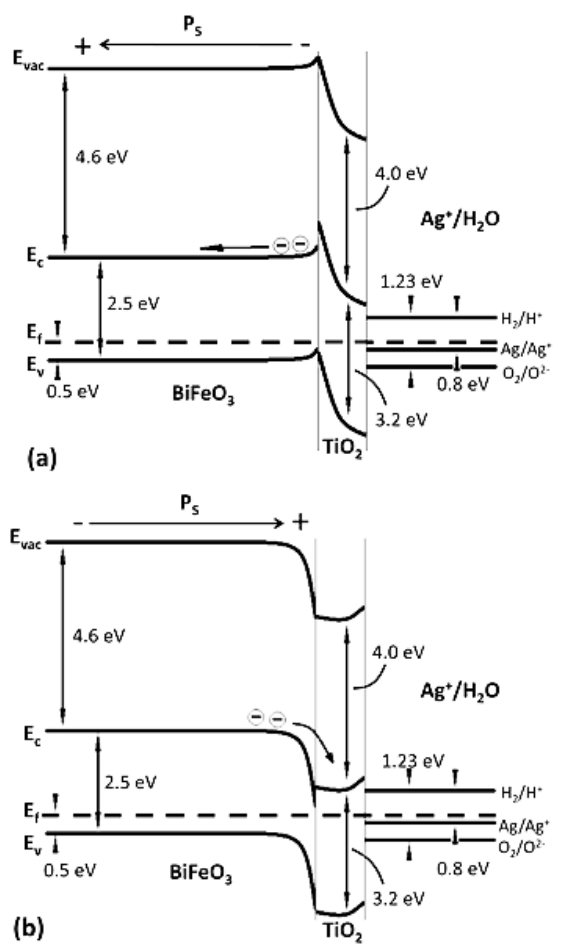

Figure 8: Schematic band structure of a $\mathrm{BiFeO}_{3} / \mathrm{TiO}_{2} / \mathrm{H}_{2} \mathrm{O}$ heterostructure with the ferroelectric polarization $\left(\mathrm{P}_{s}\right)$ of $\mathrm{BiFeO}_{3}$ (a) pointing away from the $\mathrm{BiFeO}_{3} / \mathrm{TiO}_{2}$ interface, and (b) pointing towards the $\mathrm{BiFeO}_{3} / \mathrm{TiO}_{2}$ interface. The vacuum level, conduction band edge, Fermi level and valence band edge are denoted by $\mathrm{E}_{v a c}, \mathrm{E}_{c}, \mathrm{E}_{f}$, and $\mathrm{E}_{v}$, respectively. The hydrogen, silver, and oxygen redox levels are indicated on the right. Reprinted with permission from Y. Zhang, A. M. Schultz, P. A. Salvador, and G. S. Rohrer, Journal of Materials Chemistry, 21(12), 4168-4174.

to similar monolayer systems, including $\mathrm{CrO}_{2}$ on ferroelectric $\mathrm{PbTiO}_{3}$, and a significant coupling between the monolayer chemistry and the substrate polarization was observed [10]. For electronically reconstructed surfaces, dramatic changes, in some cases in excess of $4 \mathrm{eV}$ per molecule, were seen in the binding energies of different molecules such as $\mathrm{NO}, \mathrm{NO}_{2}, \mathrm{CO}, \mathrm{CO}_{2}$ and $\mathrm{O}_{2}$ upon changing the substrate polarization direction. In addition, modifying these heterostructures with a thin $\mathrm{SrTiO}_{3}$ buffer layer between the surface monolayer and the ferroelectric substrate could stabilize them against transition metal interdiffusion and nanocrystallite formation (i.e., monolayer wetting is stabilized). In contrast to transition metals, the thickness of the $\mathrm{SrTiO}_{3}$ buffer layer has a very weak effect on the polarization dependent surface chemistry indicating that the substrate polarization propagates without hindrance through the buffer layer. This is primarily because $\mathrm{SrTiO}_{3}$ is a strongly polarizable incipient ferroelectric 
(quantum paraelectric) $[208,209]$ whose polarizability is further aided by the epitaxial strain enforced on it by the $\mathrm{PbTiO}_{3}[210]$.

On the experimental side, Inoue, et al have studied $\mathrm{O}_{2}$ and $\mathrm{H}_{2}$ adsorption on thick, in excess of $30 \mathrm{~nm}, \mathrm{NiO}$ and $\mathrm{TiO}_{2}$ films on oppositely poled $\mathrm{LiNbO}_{3}$ (0001) surfaces. For $\mathrm{NiO}$ they observed higher $\mathrm{O}_{2}$ desorption activation energies for negatively poled substrates, by $0.57 \mathrm{eV} /$ molecule, while for $\mathrm{H}_{2}$ on $\mathrm{TiO}_{2}$ they reported a $0.15 \mathrm{eV}$ per molecule higher desorption activation energy for the positively poled substrate [211]. These effects were correlated with changes in conductivity upon adsorption and were interpreted in terms similar to the field effect described above for $\mathrm{SnO}_{2}$ nanowires [201, 202]. Specifically, cation vacancies in $\mathrm{NiO}$ tend to make it a p-type wide bandgap semiconductor; on the negatively poled ferroelectric substrate, the positively charged holes are attracted to the interface, creating a band bending that facilitates charge transfer to adsorbed oxygen. An opposite effect was proposed to explain stronger adsorption of electropositive hydrogen on n-type $\mathrm{TiO}_{2}$ on the positively poled substrate. Following up on this work, Inoue and co-workers took advantage of the piezoelectric properties of ferroelectrics [212] to modulate the polarization and thus chemical properties of $\mathrm{NiO}[213,214,215]$. In these experiments, they launched surface acoustic waves ( $\mathrm{SAW}$ ) in $\mathrm{LiNbO}_{3}$ beneath $\mathrm{NiO}$ films and observed a four to seven fold increase in $\mathrm{CO}$ oxidation rates along with a decrease in the reaction activation energy. It was suggested that the temporally varying polarization induced by the $\mathrm{SAW}$ caused the $\mathrm{NiO}$ surface to alternate between strong and weak adsorption at the same sites which promoted reactant adsorption and product desorption.

For the thick films and relatively inert substrates in the experiments outlined above, the adsorbate coverages were limited to a few percent of a monolayer. Recently, thin epitaxial layers of catalytically active $\mathrm{Cr}_{2} \mathrm{O}_{3}$ layers on oppositely poled $\mathrm{LiNbO}_{3}$ (0001) substrates were prepared with the expectation that larger effects could be induced in thin layers of more reactive oxides [12]. Photoelectron spectroscopy data revealed no band offsets associated with expected interfacial dipoles or changes in the $\mathrm{Cr}$ oxidation state which was taken as evidence that the compensating charges in the form of oxygen vacancies or oxygen adatoms could migrate to the $\mathrm{Cr}$ oxide surface. Experiments, however, revealed no significant effect of substrate polarization direction on oxygen adsorption.

\subsection{Photocatalysis}

Motivated by the need to increase the stability of perovskite ferroelectrics in aqueous solutions to make them useful for photoelectrocatalysis, Burbure et al. studied the photochemical behavior of titania films on $\mathrm{BaTiO}_{3}$ substrates [216]. The reactivity was quantified by measuring the rate of sliver reduction. As with the bare ferroelectric, the reaction products were spatially distributed in patterns consistent with the ferroelectric domain structure, in this case buried beneath the non-ferroelectric film. The photochemical reactivity was characterized not only as a function of ferroelectric domain orientation but also of the titania phase and orientation [217]. Among the surfaces examined, the bare $\mathrm{BaTiO}_{3}$ substrates had the highest reactivity, $15 \mathrm{~nm}$ thick anatase films the 
next highest, while thick (110) rutile films were the least reactive. Although the bare $\mathrm{BaTiO}_{3}$ was the most active, this work demonstrated that oxide thin films can protect the ferroelectric surface against corrosion and leaching while still benefiting from the ferroelectric's enhanced electron-hole separation and spatial separation of oxidation and reduction sites.

In 2011, Zhang et al. studied the photocatalytic activity of $\mathrm{TiO}_{2} / \mathrm{BiFeO}_{3}$ heterostructures under visible light [218] also using Ag reduction as a measure of the activity. Their work explained how the band bending direction inside the $\mathrm{BiFeO}_{3}$ at the interface with $\mathrm{TiO}_{2}$ changes as a function of polarization and how this facilitates the transfer of electrons to $\mathrm{TiO}_{2}$ in positive polarization and suppresses it in the opposite polarization (see Fig. 8). Consequently, the $\mathrm{Ag}$ reduction pattern mimics the $\mathrm{BiFeO}_{3}$ domain structure. Subsequently, Li et al. studied the photocatalytic hydrogen production activity of heterostructured ceramic powders that consisted of nanostructured $\mathrm{TiO}_{2}$ surrounding microcrystalline (Ba,Sr) $\mathrm{TiO}_{3}$ cores (see Fig. 9) $[219,146]$. They demonstrated much higher hydrogen production rates for these heterostructures in water/methanol solutions than for each of the components alone; a similar result has been published on nanostructured $\mathrm{TiO}_{2}$ surrounding $\mathrm{PbTiO}_{3}$ cores [220]. A recent DFT study by Lee et al. on $\mathrm{TiO}_{2}$ /ferroelectric heterostructures also shows that the ferroelectric substrate can strongly reduce rate-limiting barriers and significantly improve the oxygen evolution reaction (OER) relative to unsupported $\mathrm{TiO}_{2}$ antase [221].

Both theory and experiment suggest that photocatalytic properties of metal oxide thin films can be significantly enhanced by a ferroelectric substrate due to band bending and charge transfer to the surface. In addition, the chemical stability of the oxide films in aqueous environments in combination with the ability to transport photogenerated carriers through the oxide makes it possible to exploit the charge separation properties of the ferroelectric to improve photocatalytic performance.

\section{Including ferroelectric switching in catalytic cycle}

In this section, we aim to provide more insight into the use of ferroelectric polarization and its coupling to strain, temperature or electric fields to enhance catalytic activity in a dynamic manner. This in turn may permit one to move beyond some of the fundamental limitations imposed on catalytic activity when considering only static and equilibrated surfaces. As discussed above, the use of ferroelectric surfaces directly or as supports can lead to interesting and enhanced surface chemistry and catalysis. A potentially interesting way to exploit ferroelectrics is to create reversible and switchable chemistry in some dynamic or cyclic manner. We note that, for many good experimental and practical reasons, most prior studies have focused on surfaces with fixed polarization states. Usually, the interesting chemistry happens for one polarization state while the opposite polarization state is used as a reference to indicate the extent of the polarization effects. Or in other cases, each polarization state is 

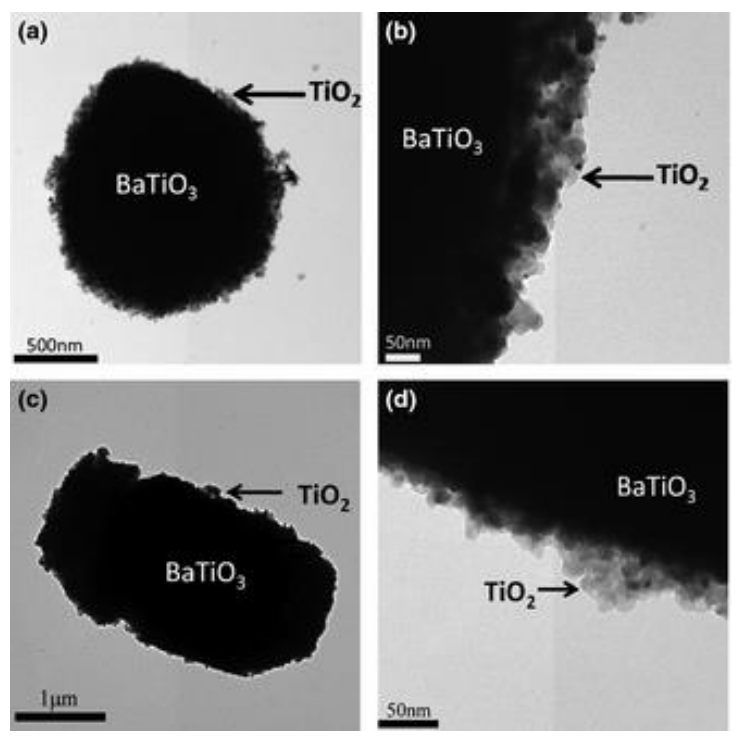

Figure 9: Bright field TEM images of a heterostructured particle composed of a microcrystalline- $\mathrm{BaTiO}_{3}$ core and nanostructured- $\mathrm{TiO}_{2}$ shell annealed at $(\mathrm{a}, \mathrm{b}) 600^{\circ} \mathrm{C}$ and (c, d) $400^{\circ} \mathrm{C}$. The entire particles are shown in $(\mathrm{a}, \mathrm{c})$, and higher magnification images of the coating are shown in (c, d). Reprinted with permission from L. Li, G. S. Rohrer, and P. A. Salvador, Journal of the American Ceramic Society, 95(4), 1414-1420.

inspected separately. Here, we discuss recent attempts to combine the activity of both polarization states in a cyclic manner.

In 2015, Kakekhani et al. proposed a cyclic catalytic scheme employing both polarization states where both are equally important parts of the cycle [10]. Beyond the creation of novel catalysts, another motivation for this work was to try to overcome some of the limitations on catalytic activity imposed by the Sabatier principle. The Sabatier principle is one of the cornerstones of catalysis [179]. It states that the efficiency of a fixed catalytic surface is maximized when the adsorbate-surface interaction strength is optimal: strong enough to drive the reactions forward but weak enough to permit the products to desorb. Hence, a large part of the effort in catalyst discovery and design has focused on fabricating and tuning material surfaces to make this interaction as optimal as possible (i.e., near the top of the ubiquitous "volcano plots") [179, 222, 223, 224, 225]. The Sabatier principle highlights an unavoidable compromise between adsorption and desorption and imposes a fundamental limitation on catalytic activity of a given, fixed surface. Kakekhani et al. proposed that, in principle, exploiting ferroelectric polarization in a cyclic manner and using both polarizations allows one to overcome some of these limitation [10]. The basic idea is to use ferroelectric polarization to switch between one surface state with strong adsorption potential and a second surface state with strong desorption potential where the two surface states are controlled by direction of the substrate 


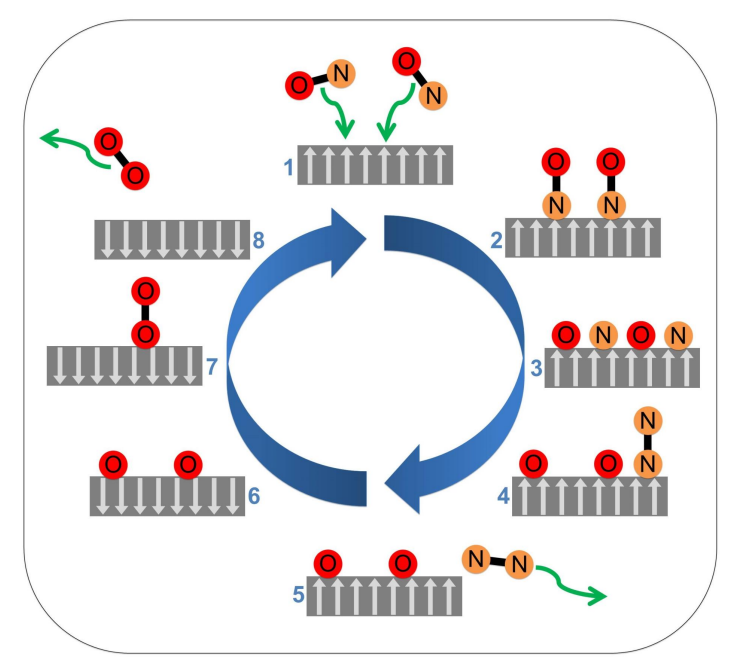

Figure 10: Proposed catalytic cycle on $\mathrm{CrO}_{2}$ monolayers supported on $\mathrm{PbTiO}_{3}$ for $\mathrm{NO}$ direct decomposition into $\mathrm{N}_{2}$ and $\mathrm{O}_{2}$. In each case, the polarization direction is indicated by gray arrows. The green arrows show adsorption and desorption of the molecules. The cycle is begun from a clean and pristine positively poled surface and through multiple steps (after effectively dissociating $2 \mathrm{NO}$ molecules into $\mathrm{N}_{2}$ and $\mathrm{O}_{2}$ ) returns to this state. Reprinted with permission from A. Kakekhani and S. Ismail-Beigi, ACS Catalysis 20155 (8), 4537-4545.

polarization (i.e., the substrate order parameter).

They considered a class of systems that consisted of a chemically active transition metal oxide $\left(\mathrm{XO}_{2}\right)$ surface monolayer (as a continuation of the ferroelectric perovskite structure) followed by a $\mathrm{SrTiO}_{3}$ (STO) buffer layer (whose thickness ranged from 1 to 5 unit cells) on top of a ferroelectric $\mathrm{PbTiO}_{3}$ substrate. Here, the STO buffer stabilizes the $\mathrm{XO}_{2}$ surface monolayer against bulk nanocrystallite formation and transition metal interdiffusion. The transition metal $\mathrm{X}$ is chosen based on the specific reaction of interest [10]. For example, their work focused on $\mathrm{NO}_{\mathrm{x}}$ direct decomposition, which has been a lasting challenge for conventional catalysis and the automotive emission control industry $[226,227,228,229,230]$, and CO oxidation. Their calculated results showed that both $\mathrm{CrO}_{2}$ and $\mathrm{RuO}_{2}$ surface monolayer could simultaneously and efficiently catalyze these two reactions when implemented as part of the proposed cyclic scheme (see Fig. 10). In this approach, the fact that ferroelectric surfaces prefer to atomically reconstruct is no longer an unfavorable or limiting factor for enhanced surface chemistry. Instead, by dynamically switching the polarization, one actually exploits the thermodynamic drive towards atomic reconstructions to drive desired reactions for each surface state. The corresponding energy landscape diagram for this cycle for the case of a $\mathrm{CrO}_{2}$ monolayer is shown in Fig. 11. Kakekhani et al. explained their computed binding energy trends by a generalization of the canonical d-band model for transition metals [231, 232, 233] 


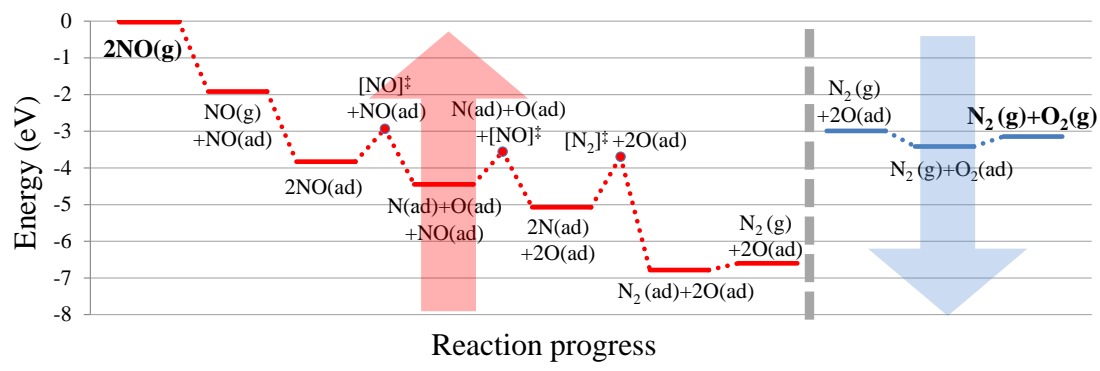

Figure 11: Energy landscape diagram for the proposed catalytic cycle on $\mathrm{CrO}_{2}$ monolayers supported on $\mathrm{PbTiO}_{3}$ for $\mathrm{NO}$ direct decomposition into $\mathrm{N}_{2}$ and $\mathrm{O}_{2}$. The two arrows indicate the direction of the polarization in each part of the cycle: on the left is positive (up) polarization (parallel to the surface normal vector), while on the right is negative (down) polarization (antiparallel to the surface normal vector). The dashed gray line indicates the point in the cycle at which the polarization is flipped. Horizontal plateaus locate local minima, circles represent transition states as highlighted by the $\ddagger$ label. The reference energy is that of two NO molecules in the gas phase above a $2 \times 2 \mathrm{CrO}_{2}-\mathrm{PbTiO}_{3}$ surface in positive polarization. Reprinted with permission from A. Kakekhani and S. Ismail-Beigi, ACS Catalysis 20155 (8), $4537-4545$.

to metal oxide surfaces combined with charge transfer effects. As pointed out by Kakekhani et al., a secondary benefit of such a scheme is that the surface periodically goes through a strong desorption phase in which the poisoning atoms and molecules (e.g., $\mathrm{SO}_{2}$ ) desorb [10]. We note that other work (presently under review) theoretically proposes cyclic enhancement of catalytic $\mathrm{CO}_{2}$ dissociation to $\mathrm{CO}$ and oxygen using ferroelectric polarization for ultrathin films of $\mathrm{ZnO}$ on $\mathrm{PbTiO}_{3}$ [115]. The experimental challenge is now to create and cycle such surface systems. The potential payoff is the creation of a switchable and/or tunable set of catalysts.

\section{Outlook and conclusions}

The effect of ferroelectric polarization on surface physics and chemistry has been studied for more than six decades [234]. The above survey of this field has shown that ferroelectric polarization can affect the adsorption energies, adsorption modes, and reaction rates of a variety of polar and non-polar molecules. These observations hold for both bare ferroelectric surfaces as well as ferroelectric substrates that support thin films of metals or metal oxides. In this final section, we take a broader view of current challenges in surface catalysis design to see how ferroelectrics may potentially contribute to overcoming a number of the challenges.

Surface catalysis based on transition metals and their alloys has been one of the most important research fields in theoretical and experimental catalysis and chemistry $[235,236]$. In addition to basic scientific discoveries, this field has had enormous impacts on daily lives, e.g., it has helped overcome the world hunger problem by introducing the Haber-Bosch process to efficiently break the 
strong $\mathrm{N}_{2}$ triple bond and synthesize ammonia which is the most important precursor for fertilizers [237, 238]. A significant breakthrough for transition metal catalysis in the last few decades has been the introduction of a predictive theory for catalysis based on a combination of the d-band model, activity maps and scaling relations $[239,240,241,242,179]$. Having efficient computational tools and a predictive theory has permitted computational scientists to design novel catalysts and computationally screen materials for a specific functionality, and then one can attempt to experimentally fabricate the promising candidates [243, 222, 244].

Among the many contributions of this descriptive theory has been the modification of the stability of $\mathrm{Ni}$ catalysts for steam reforming by the addition of gold and the mixing of cobalt and molybdenum in ammonia synthesis catalysts $[245,224,246]$. Scaling relations have been one of the foundations for understanding catalytic trends: these relations help by significantly reducing the number of potentially independent degrees of freedom (e.g., the full set of binding energies of the products and intermediates) in a chemical reaction to just a few descriptors [231, 247, 175, 248, 249, 250]. However, as Vojvodic and Norskov pointed out in a recent review, despite being extremely useful, scaling relations have also helped identify some of the limitations on the tunability of large classes of catalysts [251]. Figure 12 provides an example for the case of ammonia synthesis. Clearly, a much better catalyst for ammonia synthesis could be fabricated if one could circumvent or depart from the known scaling relations for (stepped) transition metal surfaces: the scaling relation has confined these surfaces to being far from maximally efficient. Due to this restriction, namely the reduced degrees of freedom stemming from the scaling relations, there are many reactions that cannot be fully optimized and thus suffer from inefficient catalysis. There are also some important reactions that currently have no effective catalysts (e.g., the partial oxidation of methane to methanol $[252,253,254,255]$ and the direct decomposition of $\left.\mathrm{NO}_{x}[256]\right)$. The need for an extra degree of freedom that can take us away from the constraints of the scaling relation is clear.

We suggest that ferroelectrics may make an important contribution in this regard. Ferroelectric surfaces have an added degree of freedom which is the direction of the polarization below the surface. Furthermore, the polarization couples to multiple physical degrees of freedom which permits different pathways to modulate the polarization. For example, we mentioned above how acoustic waves may modify surface electronic properties through the piezoelectric effect [213]. However, piezoelectricity is not the only possibility: temperature can modify the polarization via the pyroelectric effect or, more obviously, one can use external electric fields to directly control the polarization. We note that some ferroelectrics are multiferroic: their magnetic properties are coupled to their electrical polarization $[257,258,259]$, so that, in principle, one can also enlarge the list of external control knobs to include magnetic fields [260, 261, 262]. The overall logic is that a ferroelectric surface has added tunability because: (i) there is an order parameter (here the ferroelectric polarization) below the surface, (ii) the order parameter concerns charge degrees of freedom and thus 

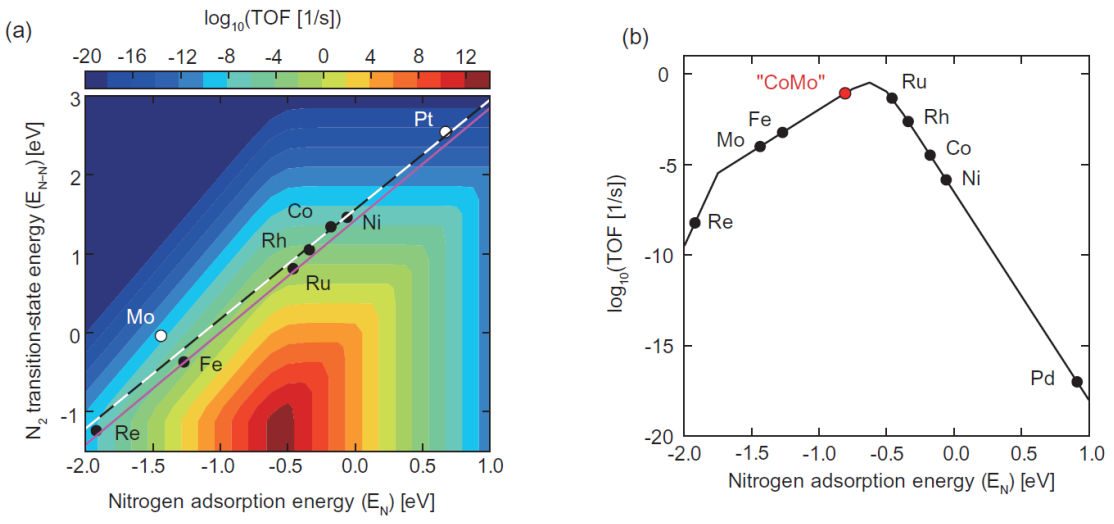

Figure 12: Ammonia synthesis rate as a function of nitrogen adsorption energy $\mathrm{E}_{\mathrm{N}}$ and $\mathrm{N}_{2}$ dissociation barrier $\mathrm{E}_{\mathrm{N}-\mathrm{N}}$ with energetics for stepped transition metal sites and the corresponding scaling relation line (dashed black-white) showing that $\mathrm{E}_{\mathrm{N}-\mathrm{N}}$ is a linear function of $\mathrm{E}_{\mathrm{N}}$, which prevents the metal steps from entering the optimum catalyst region (red area). The alkali-promoted scaling relation is given by the magenta line. b) Calculated rate as a function of a single descriptor of $E_{N}$. Reprinted with permission from A. Vojvodic and J. K. Norskov, National Science Review (2015): nwv023.

can couple strongly to the surface chemistry, and (iii) the order parameter can be controlled by external knobs. In principle, this should permit one to shift the scaling relations in their appropriate phase space.

A second benefit of using ferroelectrics is the potential for dynamic or cyclic behavior [10]. Namely, instead of simply using the polarization to shift scaling relations, one can envisage sidestepping some of the fundamental compromises in the usual approach to catalyst optimization. To reiterate a point from the previous section, the Sabatier principle enforces a compromise situation for a fixed surface: maximum activity requires an optimal adsorbate-surface interaction strength that is neither too strong nor too weak [179]. However, if one could cycle the surface between multiple states with very different interaction strengths, some of the compromises could be overcome: adsorption and desorption do not have to be competing effects but both can be periodically enhanced or suppressed to drive the appropriate set of reactions or adsorption/desorption processes in sequences [10, 115]. The SAW experiments described above provide some evidence that such a mechanism is achievable [214, 213]. We surmise that the cyclic control of ferroelectric surfaces and their chemistry may open up entirely new ways to create and design catalytic materials and processes.

\section{Acknowledgments}

The authors acknowledge the support of the National Science Foundation through grant NSF MRSEC DMR-1119826 and the Toyota Research Institute of North America. E.I.A. thanks the National Science Foundation for support 
through grant NSF CHE-1213751. Finally, the authors thank Victor E. Henrich, Jens K. Norskov, Matthew Herdiech, Alexie Kolpak, Kevin Garrity, Kimber Stamm-Masias and Paul Fanson for valuable discussions, insight and input on this topic.

[1] D. Vanderbilt, R. D. King-Smith, Electric polarization as a bulk quantity and its relation to surface charge, Phys. Rev. B 48 (7) (1993) 4442-4455.

[2] R. D. King-Smith, D. Vanderbilt, Theory of polarization of crystalline solids, Phys. Rev. B 47 (3) (1993) 1651-1654.

[3] K. Garrity, A. Kakekhani, A. Kolpak, S. Ismail-Beigi, Ferroelectric surface chemistry: First-principles study of the $\mathrm{PbTiO}_{3}$ surface, Phys. Rev. B 88 (4) (2013) 045401.

[4] C. Noguera, Polar oxide surfaces, Journal of Physics: Condensed Matter 12 (2000) R367-R410.

[5] A. Wander, N. M. Harrison, The stability of polar oxide surfaces: The interaction of $\mathrm{H} 20$ with $\mathrm{ZnO}(0001)$ and $\mathrm{ZnO}(000 \overline{1})$, The Journal of Chemical Physics 115 (5) (2001) 2312-2316.

[6] J. M. Carlsson, Electronic structure of the polar $\mathrm{ZnO}\left\{\begin{array}{llll}0 & 0 & 0 & 1\end{array}\right\}$-surfaces, Computational Materials Science 22 (1-2) (2001) 24-31.

[7] S. Sanna, R. Holscher, W. G. Schmidt, Temperature dependent LiNbO3(0 00 1): Surface reconstruction and surface charge, Applied Surface Science 301 (2014) 70-78.

[8] Y. Watanabe, M. Okano, A. Masuda, Surface Conduction on Insulating $\mathrm{BaTiO}_{3}$ Crystal Suggesting an Intrinsic Surface Electron Layer, Phys. Rev. Lett. 86 (2) (2001) 332-335.

[9] Y. Watanabe, Theoretical stability of the polarization in a thin semiconducting ferroelectric, Phys. Rev. B 57 (2) (1998) 789-804.

[10] A. Kakekhani, S. Ismail-Beigi, Ferroelectric-Based Catalysis: Switchable Surface Chemistry, ACS Catal. 5 (8) (2015) 4537-4545.

[11] Y. Sun, R. J. Nemanich, Photoinduced Ag deposition on periodically poled lithium niobate: Wavelength and polarization screening dependence, Journal of Applied Physics 109 (10) (2011) 104302.

[12] M. W. Herdiech, X. Zhu, M. D. Morales-Acosta, F. J. Walker, E. I. Altman, The modification of ferroelectric $\mathrm{LiNbO}_{3}$ (0001) surfaces using chromium oxide thin films, Phys. Chem. Chem. Phys. 17 (14) (2015) 9488-9498. 
[13] D. G. Popescu, M. A. Husanu, L. Trupina, L. Hrib, L. Pintilie, A. Barinov, S. Lizzit, P. Lacovig, C. M. Teodorescu, Spectro-microscopic photoemission evidence of charge uncompensated areas in $\mathrm{Pb}(\mathrm{Zr}, \mathrm{Ti}) \mathrm{O}_{3}$ (001) layers, Phys. Chem. Chem. Phys. 17 (1) (2015) 509-520.

[14] S. K. Streiffer, J. A. Eastman, D. D. Fong, C. Thompson, A. Munkholm, M. V. Ramana Murty, O. Auciello, G. R. Bai, G. B. Stephenson, Observation of Nanoscale $180^{\circ}$ Stripe Domains in Ferroelectric $\mathrm{PbTiO}_{3}$ Thin Films, Phys. Rev. Lett. 89 (6) (2002) 067601.

[15] S. V. Kalinin, D. A. Bonnell, Local potential and polarization screening on ferroelectric surfaces, Phys. Rev. B 63 (12) (2001) 125411.

[16] B. Meyer, First-principles study of the polar O-terminated $\mathrm{ZnO}$ surface in thermodynamic equilibrium with oxygen and hydrogen, Phys. Rev. B 69 (4) (2004) 045416.

[17] D. D. Fong, A. M. Kolpak, J. A. Eastman, S. K. Streiffer, P. H. Fuoss, G. B. Stephenson, C. Thompson, D. M. Kim, K. J. Choi, C. B. Eom, I. Grinberg, A. M. Rappe, Stabilization of Monodomain Polarization in Ultrathin $\mathrm{PbTiO}_{3}$ Films, Phys. Rev. Lett. 96 (12) (2006) 127601.

[18] Y. Xie, H.-t. Yu, G.-x. Zhang, H.-g. Fu, J.-z. Sun, First-Principles Investigation of Stability and Structural Properties of the BaTiO3 (110) Polar Surface, J. Phys. Chem. C 111 (17) (2007) 6343-6349.

[19] S. V. Levchenko, A. M. Rappe, Influence of Ferroelectric Polarization on the Equilibrium Stoichiometry of Lithium Niobate (0001) Surfaces, Phys. Rev. Lett. 100 (25) (2008) 256101.

[20] M.-H. Du, S. B. Zhang, J. E. Northrup, S. C. Erwin, Stabilization mechanisms of polar surfaces: ZnO surfaces, Phys. Rev. B 78 (15) (2008) 155424.

[21] S. Torbrugge, F. Ostendorf, M. Reichling, Stabilization of ZincTerminated $\mathrm{ZnO}(0001)$ by a Modified Surface Stoichiometry, J. Phys. Chem. C 113 (12) (2009) 4909-4914.

[22] M. Valtiner, M. Todorova, G. Grundmeier, J. Neugebauer, Temperature Stabilized Surface Reconstructions at Polar $\mathrm{ZnO(0001),} \mathrm{Phys.} \mathrm{Rev.} \mathrm{Lett.}$ 103 (6) (2009) 065502.

[23] G.-X. Zhang, Y. Xie, H.-T. Yu, H.-G. Fu, First-principles calculations of the stability and electronic properties of the PbTiO3 (110) polar surface, J. Comput. Chem. 30 (12) (2009) 1785-1798.

[24] S. Sanna, W. G. Schmidt, Lithium niobate X-cut, Y-cut, and Z-cut surfaces from ab initio theory, Phys. Rev. B 81 (21) (2010) 214116.

[25] J. Wang, G. Tang, X. S. Wu, Thermodynamic stability of BaTiO3 (110) surfaces, Phys. Status Solidi B 249 (4) (2012) 796-800. 
[26] J. L. Wang, B. Vilquin, N. Barrett, Screening of ferroelectric domains on BaTiO3(001) surface by ultraviolet photo-induced charge and dissociative water adsorption, Applied Physics Letters 101 (9) (2012) 092902.

[27] R. Wahl, J. V. Lauritsen, F. Besenbacher, G. Kresse, Stabilization mechanism for the polar $\mathrm{ZnO}(000 \overline{1})$ surface, Phys. Rev. B 87 (8) (2013) 085313.

[28] A. Calzolari, M. Bazzani, A. Catellani, Dipolar and charge transfer effects on the atomic stabilization of $\mathrm{ZnO}$ polar surfaces, Surface Science 607 (2013) 181-186.

[29] V. Staemmler, K. Fink, B. Meyer, D. Marx, M. Kunat, S. Gil Girol, U. Burghaus, C. Woll, Stabilization of Polar ZnO Surfaces: Validating Microscopic Models by Using CO as a Probe Molecule, Phys. Rev. Lett. 90 (10) (2003) 106102.

[30] M. Kunat, S. Gil Girol, T. Becker, U. Burghaus, C. Woll, Stability of the polar surfaces of $\mathrm{ZnO}$ : A reinvestigation using He-atom scattering, Phys. Rev. B 66 (8) (2002) 081402.

[31] Y. Yun, E. I. Altman, Using Ferroelectric Poling to Change Adsorption on Oxide Surfaces, J. Am. Chem. Soc. 129 (2007) 15684-15689.

[32] M. T. Kesim, J. Zhang, S. Trolier-McKinstry, J. V. Mantese, R. W. Whatmore, S. P. Alpay, Pyroelectric response of lead zirconate titanate thin films on silicon: Effect of thermal stresses, Journal of Applied Physics 114 (20) (2013) 204101.

[33] J. Karthik, L. W. Martin, Pyroelectric properties of polydomain epitaxial $\mathrm{Pb}\left(\mathrm{Zr}_{1-x} \mathrm{Ti}_{x}\right) \mathrm{O}_{3}$ thin films, Phys. Rev. B 84 (2) (2011) 024102.

[34] G. Parravano, Ferroelectric Transitions and Heterogenous Catalysis, The Journal of Chemical Physics 20 (2) (1952) 342-343.

[35] H. L. Stadler, Changing Properties of Metals by Ferroelectric Polarization Charging, Phys. Rev. Lett. 14 (24) (1965) 979-981.

[36] Y. Ishibashi, H. L. Stadler, Ferroelectric electroreflectance of gold films, Journal of Physics and Chemistry of Solids 30 (9) (1969) 2113-2116.

[37] Y. Inoue, I. Yoshioka, K. Sato, Polarization effects upon adsorptive and catalytic properties. 1. Carbon monoxide oxidation over palladium deposited on lithium niobate (LiNbO3) ferroelectrics, J. Phys. Chem. 88 (1984) 1148-1151.

[38] Y. Inoue, K. Sato, S. Suzuki, Polarization effects upon adsorptive and catalytic properties. 2. Surface electrical conductivity of nickel(II) oxide deposited on lithium niobate (LiNbO3) and its changes upon gas adsorption, J. Phys. Chem. 89 (1985) 2827-2831. 
[39] S. Akhter, K. Lui, H. H. Kung, Comparison of the chemical properties of the zinc-polar, the oxygen-polar, and the nonpolar surfaces of zinc oxide, J. Phys. Chem. 89 (1985) 1958-1964.

[40] J. M. Vohs, M. A. Barteau, Conversion of methanol, formaldehyde and formic acid on the polar faces of zinc oxide, Surface Science 176 (1-2) (1986) 91-114.

[41] Y. Inoue, M. Okamura, K. Sato, A thin-film semiconducting titanium dioxide combined with ferroelectrics for photoassisted water decomposition, J. Phys. Chem. 89 (1985) 5184-5187.

[42] Y. Inoue, K. Sato, K. Sato, H. Miyama, Photoassisted water decomposition by ferroelectric lead zirconate titanate ceramics with anomalous photovoltaic effects, J. Phys. Chem. 90 (1986) 2809-2810.

[43] Y. Inoue, K. Sato, K. Sato, H. Miyama, A device type photocatalyst using oppositely polarized ferroelectric substrates, Chemical Physics Letters 129 (1) (1986) 79-81.

[44] Y. Inoue, K. Sato, K. Sato, Photovoltaic and photocatalytic behaviour of a ferroelectric semiconductor, lead strontium zirconate titanate, with a polarization axis perpendicular to the surface, Journal of the Chemical Society, Faraday Transactions 1: Physical Chemistry in Condensed Phases 85 (7) (1989) 1765.

[45] Y. Inoue, O. Hayashi, K. Sato, Photocatalytic activities of potassiumdoped lead niobates and the effect of poling, J. Chem. Soc., Faraday Trans. 86 (12) (1990) 2277-2282.

[46] W. Ji, K. Yao, Y.-F. Lim, Y. C. Liang, A. Suwardi, Epitaxial ferroelectric $\mathrm{BiFeO} 3$ thin films for unassisted photocatalytic water splitting, Applied Physics Letters 103 (6) (2013) 062901.

[47] X. Cheng, W. Dong, F. Zheng, L. Fang, M. Shen, Enhanced photocathodic behaviors of $\mathrm{Pb}(\mathrm{Zr0}$.20ti0.80)O3 films on Si substrates for hydrogen production, Applied Physics Letters 106 (24) (2015) 243901.

[48] R. Li, Q. Li, L. Zong, X. Wang, J. Yang, BaTiO3/TiO2 heterostructure nanotube arrays for improved photoelectrochemical and photocatalytic activity, Electrochimica Acta 91 (2013) 30-35.

[49] C. Zhen, J. C. Yu, G. Liu, H.-M. Cheng, Selective deposition of redox co-catalyst(s) to improve the photocatalytic activity of single-domain ferroelectric $\mathrm{PbTiO}_{3}$ nanoplates, Chemical Communications 50 (72) (2014) 10416.

[50] J. L. Giocondi, G. S. Rohrer, The Influence of the Dipolar Field Effect on the Photochemical Reactivity of Sr2nb2o7 and BaTiO3 Microcrystals, Top Catal 49 (1-2) (2008) 18-23. 
[51] N. V. Burbure, P. A. Salvador, G. S. Rohrer, Influence of Dipolar Fields on the Photochemical Reactivity of Thin Titania Films on BaTiO3 Substrates, Journal of the American Ceramic Society 89 (9) (2006) 2943-2945.

[52] Z. Shen, X. Qu, Y. Chen, R. Liu, Nanoembossing Induced Ferroelectric Lithography on PZT Films for Silver Particle Patterning, ACS Nano 5 (9) (2011) 6855-6860.

[53] S. Mohan, B. Subramanian, I. Bhaumik, P. K. Gupta, S. N. Jaisankar, Nanostructured $\mathrm{Bi}(1-\mathrm{x}) \mathrm{Gd}(\mathrm{x}) \mathrm{FeO} 3$ - a multiferroic photocatalyst on its sunlight driven photocatalytic activity, RSC Advances 4 (32) (2014) 16871.

[54] Q. Li, R. Li, L. Zong, J. He, X. Wang, J. Yang, Photoelectrochemical and photocatalytic properties of Ag-loaded $\mathrm{BaTiO} 3 / \mathrm{TiO} 2$ heterostructure nanotube arrays, International Journal of Hydrogen Energy 38 (29) (2013) 12977-12983.

[55] G. Naresh, T. K. Mandal, Excellent Sun-Light-Driven Photocatalytic Activity by Aurivillius Layered Perovskites, Bi5-xLaxTi3feo15 ( $\mathrm{x}=1,2)$, ACS Appl. Mater. Interfaces 6 (23) (2014) 21000-21010.

[56] R. Munprom, P. A. Salvador, G. S. Rohrer, Ferroelastic domains improve photochemical reactivity: a comparative study of monoclinic and tetragonal $\left(\mathrm{Bi}_{1-0.5 \mathrm{x}} \mathrm{Na}_{0.5 \mathrm{x}}\right)\left(\mathrm{V}_{1-\mathrm{x}} \mathrm{Mo}_{\mathrm{x}}\right) \mathrm{O}_{4}$ ceramics, J. Mater. Chem. A.

[57] M. Stock, S. Dunn, Linbo3 - A new material for artificial photosynthesis, IEEE Transactions on Ultrasonics, Ferroelectrics, and Frequency Control 58 (9) (2011) 1988-1993.

[58] R. K. Nath, M. F. M. Zain, A. A. H. Kadhum, Artificial Photosynthesis using LiNbO3 as Photocatalyst for Sustainable and Environmental Friendly Construction and Reduction of Global Warming: A Review, Catalysis Reviews 56 (2) (2014) 175-186.

[59] C. R. Bowen, H. A. Kim, P. M. Weaver, S. Dunn, Piezoelectric and ferroelectric materials and structures for energy harvesting applications, Energy Environ. Sci. 7 (1) (2014) 25-44.

[60] M. Batzill, Fundamental aspects of surface engineering of transition metal oxide photocatalysts, Energy \& Environmental Science 4 (9) (2011) 3275.

[61] J. L. Giocondi, G. S. Rohrer, Spatially Selective Photochemical Reduction of Silver on the Surface of Ferroelectric Barium Titanate, Chem. Mater. 13 (2001) 241-242.

[62] J. L. Giocondi, G. S. Rohrer, Spatial Separation of Photochemical Oxidation and Reduction Reactions on the Surface of Ferroelectric BaTiO3, J. Phys. Chem. B 105 (35) (2001) 8275-8277. 
[63] S. V. Kalinin, D. A. Bonnell, T. Alvarez, X. Lei, Z. Hu, J. H. Ferris, Q. Zhang, S. Dunn, Atomic Polarization and Local Reactivity on Ferroelectric Surfaces: A New Route toward Complex Nanostructures, Nano Lett. 2 (6) (2002) 589-593.

[64] S. V. Kalinin, D. A. Bonnell, T. Alvarez, X. Lei, Z. Hu, R. Shao, J. H. Ferris, Ferroelectric Lithography of Multicomponent Nanostructures, Advanced Materials 16 (910) (2004) 795-799.

[65] D. Li, D. A. Bonnell, Ferroelectric lithography, Ceramics International 34 (1) (2008) 157-164.

[66] D. Conklin, T.-H. Park, S. Nanayakkara, M. J. Therien, D. A. Bonnell, Controlling Polarization Dependent Reactions to Fabricate MultiComponent Functional Nanostructures, Adv. Funct. Mater. 21 (24) (2011) $4712-4718$.

[67] J. N. Hanson, B. J. Rodriguez, R. J. Nemanich, A. Gruverman, Fabrication of metallic nanowires on a ferroelectric template via photochemical reaction, Nanotechnology 17 (2006) 4946-4949.

[68] K. Terabe, M. Nakamura, S. Takekawa, K. Kitamura, S. Higuchi, Y. Gotoh, Y. Cho, Microscale to nanoscale ferroelectric domain and surface engineering of a near-stoichiometric LiNbO3 crystal, Applied Physics Letters 82 (3) (2003) 433-435.

[69] L. Balobaid, N. C. Carville, M. Manzo, K. Gallo, B. J. Rodriguez, Direct shape control of photoreduced nanostructures on proton exchanged ferroelectric templates, Applied Physics Letters 102 (4) (2013) 042908.

[70] C. R. Bowen, J. Taylor, E. LeBoulbar, D. Zabek, A. Chauhan, R. Vaish, Pyroelectric materials and devices for energy harvesting applications, Energy Environ. Sci. 7 (12) (2014) 3836-3856.

[71] G. Sebald, E. Lefeuvre, D. Guyomar, Pyroelectric energy conversion: Optimization principles, IEEE Transactions on Ultrasonics, Ferroelectrics, and Frequency Control 55 (3) (2008) 538-551. doi:10.1109/TUFFC.2008.680.

[72] J. D. Brownridge, S. M. Shafroth, Self-focused electron beams produced by pyroelectric crystals on heating or cooling in dilute gases, Applied Physics Letters 79 (20) (2001) 3364-3366.

[73] B. Naranjo, J. K. Gimzewski, S. Putterman, Observation of nuclear fusion driven by a pyroelectric crystal, Nature 434 (7037) (2005) 1115-1117.

[74] J. Geuther, Y. Danon, F. Saglime, Nuclear Reactions Induced by a Pyroelectric Accelerator, Phys. Rev. Lett. 96 (5) (2006) 054803. 
[75] J. A. Geuther, Y. Danon, Enhanced neutron production from pyroelectric fusion, Applied Physics Letters 90 (17) (2007) 174103.

[76] F. Merola, S. Grilli, S. Coppola, V. Vespini, S. De Nicola, P. Maddalena, C. Carfagna, P. Ferraro, Reversible Fragmentation and Self-Assembling of Nematic Liquid Crystal Droplets on Functionalized Pyroelectric Substrates, Adv. Funct. Mater. 22 (15) (2012) 3267-3272.

[77] X. Xi, D. Zhao, F. Tong, T. Cao, The self-assembly and patterning of thin polymer films on pyroelectric substrates driven by electrohydrodynamic instability, Soft Matter 8 (2) (2012) 298-302.

[78] V. Y. Shur, D. K. Kuznetsov, E. A. Mingaliev, E. M. Yakunina, A. I. Lobov, A. V. Ievlev, In situ investigation of formation of self-assembled nanodomain structure in lithium niobate after pulse laser irradiation, Applied Physics Letters 99 (8) (2011) 082901.

[79] P. Ferraro, S. Grilli, L. Miccio, V. Vespini, Wettability patterning of lithium niobate substrate by modulating pyroelectric effect to form microarray of sessile droplets, Applied Physics Letters 92 (21) (2008) 213107.

[80] O. Gennari, S. Grilli, S. Coppola, V. Pagliarulo, V. Vespini, G. Coppola, S. Bhowmick, M. A. Gioffre, G. Gentile, V. Ambrogi, P. Cerruti, C. Carfagna, P. Ferraro, Spontaneous Assembly of Carbon-Based Chains in Polymer Matrixes through Surface Charge Templates, Langmuir 29 (50) (2013) 15503-15510.

[81] E. Gutmann, A. Benke, K. Gerth, H. Bottcher, E. Mehner, C. Klein, U. Krause-Buchholz, U. Bergmann, W. Pompe, D. C. Meyer, Pyroelectrocatalytic Disinfection Using the Pyroelectric Effect of Nano- and Microcrystalline LiNbO3 and LiTaO3 Particles, J. Phys. Chem. C 116 (9) (2012) 5383-5393.

[82] B. Xiao, V. Avrutin, H. Liu, U. Ozgur, H. Morkoc, C. Lu, Large pyroelectric effect in undoped epitaxial $\mathrm{Pb}(\mathrm{Zr}, \mathrm{Ti}) \mathrm{O} 3$ thin films on $\mathrm{SrTiO} 3$ substrates, Applied Physics Letters 93 (5) (2008) 052913.

[83] S. Grilli, L. Miccio, V. Vespini, A. Finizio, S. De Nicola, P. Ferraro, Liquid micro-lens array activated by selective electrowetting on lithium niobate substrates, Optics Express 16 (11) (2008) 8084.

[84] P. Giannozzi, S. Baroni, N. Bonini, M. Calandra, R. Car, C. Cavazzoni, D. Ceresoli, G. L. Chiarotti, M. Cococcioni, I. Dabo, A. Dal Corso, S. de Gironcoli, S. Fabris, G. Fratesi, R. Gebauer, U. Gerstmann, C. Gougoussis, A. Kokalj, M. Lazzeri, L. Martin-Samos, N. Marzari, F. Mauri, R. Mazzarello, S. Paolini, A. Pasquarello, L. Paulatto, C. Sbraccia, S. Scandolo, G. Sclauzero, A. P. Seitsonen, A. Smogunov, P. Umari, R. M. Wentzcovitch, QUANTUM ESPRESSO: a modular and open-source software project for quantum simulations of materials, Journal of Physics: Condensed Matter 21 (39) (2009) 395502. 
[85] P. Hohenberg, W. Kohn, Inhomogeneous Electron Gas, Phys. Rev. 136 (3B) (1964) B864-B871.

[86] W. Kohn, L. J. Sham, Self-Consistent Equations Including Exchange and Correlation Effects, Phys. Rev. 140 (4A) (1965) A1133-A1138.

[87] J. P. Perdew, K. Burke, M. Ernzerhof, Generalized Gradient Approximation Made Simple, Phys. Rev. Lett. 77 (18) (1996) 3865-3868.

[88] J. P. Perdew, A. Zunger, Self-interaction correction to density-functional approximations for many-electron systems, Phys. Rev. B 23 (10) (1981) $5048-5079$.

[89] B. Hammer, L. B. Hansen, J. K. Norskov, Improved adsorption energetics within density-functional theory using revised Perdew-Burke-Ernzerhof functionals, Phys. Rev. B 59 (11) (1999) 7413-7421.

[90] J. Wellendorff, K. T. Lundgaard, A. Mogelhoj, V. Petzold, D. D. Landis, J. K. Norskov, T. Bligaard, K. W. Jacobsen, Density functionals for surface science: Exchange-correlation model development with Bayesian error estimation, Phys. Rev. B 85 (23) (2012) 235149.

[91] A. Pojani, F. Finocchi, C. Noguera, Polarity on the SrTiO3 (111) and (110) surfaces, Surface Science 442 (2) (1999) 179-198.

[92] C. Thierfelder, S. Sanna, A. Schindlmayr, W. G. Schmidt, Do we know the band gap of lithium niobate?, Phys. Status Solidi (c) 7 (2) (2010) $362-365$.

[93] S. Piskunov, E. Heifets, R. I. Eglitis, G. Borstel, Bulk properties and electronic structure of $\mathrm{SrTiO} 3, \mathrm{BaTiO} 3, \mathrm{PbTiO} 3$ perovskites: an ab initio HF/DFT study, Computational Materials Science 29 (2) (2004) 165-178.

[94] S. Piskunov, E. A. Kotomin, E. Heifets, J. Maier, R. I. Eglitis, G. Borstel, Hybrid DFT calculations of the atomic and electronic structure for ABO3 perovskite $\left(\begin{array}{lll}0 & 0 & 1\end{array}\right)$ surfaces, Surface Science 575 (1-2) (2005) 75-88.

[95] J. Yang, J. Long, L. Yang, First-principles investigations of the physical properties of lithium niobate and lithium tantalate, Physica B: Condensed Matter 425 (2013) 12-16.

[96] S. Sanna, C. Thierfelder, S. Wippermann, T. P. Sinha, W. G. Schmidt, Barium titanate ground- and excited-state properties from first-principles calculations, Phys. Rev. B 83 (5) (2011) 054112.

[97] R. V. Wang, D. D. Fong, F. Jiang, M. J. Highland, P. H. Fuoss, C. Thompson, A. M. Kolpak, J. A. Eastman, S. K. Streiffer, A. M. Rappe, G. B. Stephenson, Reversible Chemical Switching of a Ferroelectric Film, Phys. Rev. Lett. 102 (4) (2009) 047601. 
[98] Y. Mi, G. Geneste, J. E. Rault, C. Mathieu, A. Pancotti, N. Barrett, Polarization dependent chemistry of ferroelectric BaTiO3(001) domains, J. Phys.: Condens. Matter 24 (27) (2012) 275901.

[99] S. Cherifi, R. Hertel, S. Fusil, H. Bea, K. Bouzehouane, J. Allibe, M. Bibes, A. Barthelemy, Imaging ferroelectric domains in multiferroics using a lowenergy electron microscope in the mirror operation mode, phys. stat. sol. (RRL) 4 (1-2) (2010) 22-24.

[100] T. Kubo, H. Nozoye, Surface structure of SrTiO3(1 0 0), Surface Science 542 (3) (2003) 177-191.

[101] A. M. Kolpak, D. Li, R. Shao, A. M. Rappe, D. A. Bonnell, Evolution of the Structure and Thermodynamic Stability of the $\mathrm{BaTiO}_{3}$ (001) Surface, Phys. Rev. Lett. 101 (3) (2008) 036102.

[102] K. Reuter, M. Scheffler, First-Principles Atomistic Thermodynamics for Oxidation Catalysis: Surface Phase Diagrams and Catalytically Interesting Regions, Phys. Rev. Lett. 90 (4) (2003) 046103.

[103] P. Chen, Y. Xu, N. Wang, A. R. Oganov, W. Duan, Effects of ferroelectric polarization on surface phase diagram: Evolutionary algorithm study of the $\mathrm{BaTiO}_{3}(001)$ surface, Phys. Rev. B 92 (8) (2015) 085432.

[104] A. Y. Lushkin, V. B. Nazarenko, K. N. Pilipchak, V. F. Shnyukov, A. G. Naumovets, The impact of annealing and evaporation of crystals on their surface composition, Journal of Physics D: Applied Physics 32 (1999) 915.

[105] Y. Yun, M. Li, D. Liao, L. Kampschulte, E. I. Altman, Geometric and electronic structure of positively and negatively poled LiNbO3 $\left(\begin{array}{llll}0 & 0 & 0 & 1\end{array}\right)$ surfaces, Surface Science 601 (19) (2007) 4636-4647.

[106] H. Kawanowa, R. Souda, H. Ozawa, Y. Gotoh, K. Terabe, S. Takekawa, K. Kitamura, Structure analysis of stoichiometric LiNbO3( $\left.\begin{array}{llll}0 & 0 & 1\end{array}\right)$ surfaces using low-energy neutral scattering spectroscopy, Surface Science 538 (3) (2003) L500-L504.

[107] F. J. Giessibl, H. Bielefeldt, Physical interpretation of frequencymodulation atomic force microscopy, Phys. Rev. B 61 (15) (2000) 99689971.

[108] T. R. Albrecht, P. Grutter, D. Horne, D. Rugar, Frequency modulation detection using high-Q cantilevers for enhanced force microscope sensitivity, Journal of Applied Physics 69 (2) (1991) 668-673.

[109] T. Fukuma, K. Kobayashi, K. Matsushige, H. Yamada, True atomic resolution in liquid by frequency-modulation atomic force microscopy, Applied Physics Letters 87 (3) (2005) 034101. 
[110] S. Sanna, S. Rode, R. Holscher, S. Klassen, C. Marutschke, K. Kobayashi, H. Yamada, W. G. Schmidt, A. Kuhnle, Charge compensation by longperiod reconstruction in strongly polar lithium niobate surfaces, Phys. Rev. B 88 (11) (2013) 115422.

[111] S. Sanna, C. Dues, W. G. Schmidt, Modeling atomic force microscopy at LiNbO3 surfaces from first-principles, Computational Materials Science 103 (2015) 145-150.

[112] E. Arenholz, G. van der Laan, A. Fraile-Rodriguez, P. Yu, Q. He, R. Ramesh, Probing ferroelectricity in $\mathrm{PbZr}_{0.2} \mathrm{Ti}_{0.8} \mathrm{O}_{3}$ with polarized soft x rays, Phys. Rev. B 82 (14) (2010) 140103.

[113] R. Holscher, W. G. Schmidt, S. Sanna, Modeling LiNbO3 Surfaces at Ambient Conditions, J. Phys. Chem. C 118 (19) (2014) 10213-10220.

[114] S. Kim, M. R. Schoenberg, A. M. Rappe, Polarization Dependence of Palladium Deposition on Ferroelectric Lithium Niobate (0001) Surfaces, Phys. Rev. Lett. 107 (7) (2011) 076102.

[115] B. Alawode, A. Kolpak, $\mathrm{PbTiO}_{3}(001)$ Capped with $\mathrm{ZnO}(11 \overline{2} 0)$ : An Ab-Initio Study of Effect of Substrate Polarization on Interface Composition and $\mathrm{CO}_{2}$ Dissociation, arXiv:1507.07282 [cond-mat, physics:physics]ArXiv: 1507.07282.

[116] S. Hovel, C. Kolczewski, M. Wuhn, J. Albers, K. Weiss, V. Staemmler, C. Woll, Pyridine adsorption on the polar $\mathrm{ZnO}(0001)$ surface: $\mathrm{Zn}$ termination versus $\mathrm{O}$ termination, The Journal of Chemical Physics 112 (8) (2000) 3909-3916.

[117] B. Meyer, D. Marx, First-principles study of CO adsorption on $\mathrm{ZnO}$ surfaces, Journal of Physics: Condensed Matter 15 (2003) L89-L94.

[118] K. Nishidate, M. Yoshizawa, M. Hasegawa, Energetics of Mg and B adsorption on polar zinc oxide surfaces from first principles, Phys. Rev. B 77 (3) (2008) 035330.

[119] M. Losurdo, M. M. Giangregorio, Interaction of atomic hydrogen with Zn-polar and O-polar ZnO surfaces, Applied Physics Letters 86 (9) (2005) 091901.

[120] X. Sun, Y. J. Su, X. Li, K. W. Gao, L. J. Qiao, Stability of nano-scale ferroelectric domains in a LiNbO3 single crystal: The role of surface energy and polar molecule adsorption, Journal of Applied Physics 111 (9) (2012) 094110 .

[121] B. Jiang, Y. Bai, W. Y. Chu, S. Q. Shi, L. J. Qiao, Y. J. Su, Effect of humidity on domain switching behaviors of $\mathrm{BaTiO} 3$ single crystal under sustained load, Applied Surface Science 254 (17) (2008) 5594-5598. 
[122] R. Ferris, B. Yellen, S. Zauscher, Ferroelectric Thin Films in Fluidic Environments: A New Interface for Sensing and Manipulation of Matter, Small 8 (1) (2012) 28-35.

[123] Y. Yun, L. Kampschulte, M. Li, D. Liao, E. I. Altman, Effect of Ferroelectric Poling on the Adsorption of 2-Propanol on LiNbO3(0001), J. Phys. Chem. C 111 (37) (2007) 13951-13956.

[124] M. W. Herdiech, H. Monig, E. I. Altman, An X-ray photoelectron spectroscopy study of BF3 adsorption on positively and negatively poled LiNbO3 (0001), Surface Science 626 (2014) 53-60.

[125] Z. Zhang, R. Gonzalez, G. Diaz, L. G. Rosa, I. Ketsman, X. Zhang, P. Sharma, A. Gruverman, P. A. Dowben, Polarization Mediated Chemistry on Ferroelectric Polymer Surfaces, J. Phys. Chem. C 115 (26) (2011) 13041-13046.

[126] Z. Zhang, P. Sharma, C. N. Borca, P. A. Dowben, A. Gruverman, Polarization-specific adsorption of organic molecules on ferroelectric LiNbO3 surfaces, Applied Physics Letters 97 (24) (2010) 243702.

[127] J. Garra, J. M. Vohs, D. A. Bonnell, The effect of ferroelectric polarization on the interaction of water and methanol with the surface of LiNbO3(0 0 0 1), Surface Science 603 (8) (2009) 1106-1114.

[128] S. Sanna, R. Holscher, W. G. Schmidt, Polarization-dependent water adsorption on the $\mathrm{LiNbO}_{3}(0001)$ surface, Phys. Rev. B 86 (20) (2012) 205407.

[129] M. H. Zhao, D. A. Bonnell, J. M. Vohs, Influence of ferroelectric polarization on the energetics of the reaction of 2-fluoroethanol on BaTiO3, Surface Science 603 (2) (2009) 284-290.

[130] D. Li, M. H. Zhao, J. Garra, A. M. Kolpak, A. M. Rappe, D. A. Bonnell, J. M. Vohs, Direct in situ determination of the polarization dependence of physisorption on ferroelectric surfaces, Nat Mater 7 (6) (2008) 473-477.

[131] M. H. Zhao, D. A. Bonnell, J. M. Vohs, Effect of ferroelectric polarization on the adsorption and reaction of ethanol on BaTiO3, Surface Science 602 (17) (2008) 2849-2855.

[132] L. E. Stoflea, N. G. Apostol, L. Trupina, C. M. Teodorescu, Selective adsorption of contaminants on $\mathrm{Pb}(\mathrm{Zr}, \mathrm{Ti}) \mathrm{O}_{3}$ surfaces shown by X-ray photoelectron spectroscopy, Journal of Materials Chemistry A 2 (35) (2014) 14386 .

[133] C. Braun, S. Sanna, W. G. Schmidt, Liquid Crystal (8cb) Molecular Adsorption on Lithium Niobate Z-Cut Surfaces, J. Phys. Chem. C 119 (17) (2015) 9342-9346. 
[134] A. Riefer, S. Sanna, W. G. Schmidt, Polarization-dependent methanol adsorption on lithium niobate Z-cut surfaces, Phys. Rev. B 86 (12) (2012) 125410 .

[135] P. M. Jones, D. E. Gallardo, S. Dunn, Photochemical Investigation of a Polarizable Semiconductor, Lead-Zirconate-Titanate, Chem. Mater. 20 (18) (2008) 5901-5906.

[136] D. Arney, T. Watkins, P. A. Maggard, Effects of Particle Surface Areas and Microstructures on Photocatalytic $\mathrm{H} 2$ and $\mathrm{O} 2$ Production over $\mathrm{Pb}-$ TiO3, Journal of the American Ceramic Society 94 (5) (2011) 1483-1489.

[137] K. Lau, Y. Liu, Q. Li, Z. Li, R. L. Withers, Z. Xu, Domain-selective photochemical reaction on oriented ferroelectric $\mathrm{Pb}(\mathrm{In} 1 / 2 \mathrm{nb} 1 / 2) \mathrm{O} 3-\mathrm{Pb}(\mathrm{Mg} 1 / 3 \mathrm{nb} 2 / 3) \mathrm{O} 3-\mathrm{PbTiO} 3$ single crystals, Applied Surface Science 265 (2013) 157-161.

[138] M. Zhang, C. Jiang, W. Dong, F. Zheng, L. Fang, X. Su, M. Shen, Composition dependence of the photochemical reduction of $\mathrm{Ag}^{+}$by as-grown $\mathrm{Pb}\left(\mathrm{Zr}_{\mathrm{x}} \mathrm{Ti}_{1-\mathrm{x}}\right) \mathrm{O}_{3}$ films on indium tin oxide electrode, Applied Physics Letters 103 (10) (2013) 102902.

[139] J.-H. Kim, W. Yang, selective growth of Au nanoparticles on PZT substrates by using a photochemical reaction, Journal of the Korean Physical Society 60 (10) (2012) 1798-1802.

[140] M. Rioult, S. Datta, D. Stanescu, S. Stanescu, R. Belkhou, F. Maccherozzi, H. Magnan, A. Barbier, Tailoring the photocurrent in BaTiO3/Nb:SrTiO3 photoanodes by controlled ferroelectric polarization, Applied Physics Letters 107 (10) (2015) 103901.

[141] A. M. Schultz, Y. Zhang, P. A. Salvador, G. S. Rohrer, Effect of Crystal and Domain Orientation on the Visible-Light Photochemical Reduction of $\mathrm{Ag}$ on BiFeO3, ACS Appl. Mater. Interfaces 3 (5) (2011) 1562-1567.

[142] A. Bhardwaj, N. V. Burbure, A. Gamalski, G. S. Rohrer, Composition Dependence of the Photochemical reduction of $\mathrm{Ag}$ by Ba1-xSrxTiO3, Chem. Mater. 22 (11) (2010) 3527-3534.

[143] T. M. Shaw, S. Trolier-McKinstry, P. C. McIntyre, The Properties of Ferroelectric Films at Small Dimensions, Annual Review of Materials Science 30 (1) (2000) 263-298.

[144] C.-J. Chen, C.-H. Liao, K.-C. Hsu, Y.-T. Wu, J. C. S. Wu, P-N junction mechanism on improved $\mathrm{NiO} / \mathrm{TiO} 2$ photocatalyst, Catalysis Communications 12 (14) (2011) 1307-1310.

[145] W. Wang, X. Huang, S. Wu, Y. Zhou, L. Wang, H. Shi, Y. Liang, B. Zou, Preparation of p-n junction $\mathrm{Cu} 2 \mathrm{o} / \mathrm{BiVO} 4$ heterogeneous nanostructures with enhanced visible-light photocatalytic activity, Applied Catalysis B: Environmental 134-135 (2013) 293-301. 
[146] L. Li, P. A. Salvador, G. S. Rohrer, Photocatalysts with internal electric fields, Nanoscale 6 (1) (2014) 24-42.

[147] S. Park, C. W. Lee, M.-G. Kang, S. Kim, H. J. Kim, J. E. Kwon, S. Y. Park, C.-Y. Kang, K. S. Hong, K. T. Nam, A ferroelectric photocatalyst for enhancing hydrogen evolution: polarized particulate suspension, Physical Chemistry Chemical Physics 16 (22) (2014) 10408.

[148] Y. Yuan, Z. Xiao, B. Yang, J. Huang, Arising applications of ferroelectric materials in photovoltaic devices, J. Mater. Chem. A 2 (17) (2014) 60276041 .

[149] S. Y. Yang, L. W. Martin, S. J. Byrnes, T. E. Conry, S. R. Basu, D. Paran, L. Reichertz, J. Ihlefeld, C. Adamo, A. Melville, Y.-H. Chu, C.-H. Yang, J. L. Musfeldt, D. G. Schlom, J. W. A. Iii, R. Ramesh, Photovoltaic effects in BiFeO3, Applied Physics Letters 95 (6) (2009) 062909.

[150] H. Huang, Solar energy: Ferroelectric photovoltaics, Nat Photon 4 (3) (2010) 134-135.

[151] S. Y. Yang, J. Seidel, S. J. Byrnes, P. Shafer, C.-H. Yang, M. D. Rossell, P. Yu, Y.-H. Chu, J. F. Scott, J. W. Ager, L. W. Martin, R. Ramesh, Above-bandgap voltages from ferroelectric photovoltaic devices, Nat Nano 5 (2) (2010) 143-147.

[152] J. Seidel, D. Fu, S.-Y. Yang, E. Alarcon-Llado, J. Wu, R. Ramesh, J. W. Ager, Efficient Photovoltaic Current Generation at Ferroelectric Domain Walls, Phys. Rev. Lett. 107 (12) (2011) 126805.

[153] S. M. Young, F. Zheng, A. M. Rappe, First-Principles Calculation of the Bulk Photovoltaic Effect in Bismuth Ferrite, Phys. Rev. Lett. 109 (23) (2012) 236601.

[154] J. Kreisel, M. Alexe, P. A. Thomas, A photoferroelectric material is more than the sum of its parts, Nat Mater 11 (4) (2012) 260-260.

[155] R. Guo, L. You, Y. Zhou, Z. Shiuh Lim, X. Zou, L. Chen, R. Ramesh, J. Wang, Non-volatile memory based on the ferroelectric photovoltaic effect, Nat Commun 4.

[156] A. Bhatnagar, A. Roy Chaudhuri, Y. Heon Kim, D. Hesse, M. Alexe, Role of domain walls in the abnormal photovoltaic effect in BiFeO3, Nat Commun 4.

[157] I. Grinberg, D. V. West, M. Torres, G. Gou, D. M. Stein, L. Wu, G. Chen, E. M. Gallo, A. R. Akbashev, P. K. Davies, J. E. Spanier, A. M. Rappe, Perovskite oxides for visible-light-absorbing ferroelectric and photovoltaic materials, Nature 503 (7477) (2013) 509-512. 
[158] T. Choi, S. Lee, Y. J. Choi, V. Kiryukhin, S.-W. Cheong, Switchable Ferroelectric Diode and Photovoltaic Effect in BiFeO3, Science 324 (5923) (2009) 63-66.

[159] H. T. Yi, T. Choi, S. G. Choi, Y. S. Oh, S.-W. Cheong, Mechanism of the Switchable Photovoltaic Effect in Ferroelectric BiFeO3, Adv. Mater. 23 (30) (2011) 3403-3407.

[160] B. Chen, Z. Zuo, Y. Liu, Q.-F. Zhan, Y. Xie, H. Yang, G. Dai, Z. Li, G. Xu, R.-W. Li, Tunable photovoltaic effects in transparent

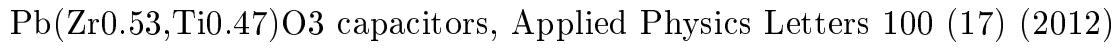
173903.

[161] R. K. Katiyar, P. Misra, F. Mendoza, G. Morell, R. S. Katiyar, Switchable photovoltaic effect in bilayer graphene/BiFeO3/Pt heterostructures, Applied Physics Letters 105 (14) (2014) 142902.

[162] R. K. Katiyar, Y. Sharma, P. Misra, V. S. Puli, S. Sahoo, A. Kumar, J. F. Scott, G. Morell, B. R. Weiner, R. S. Katiyar, Studies of the switchable photovoltaic effect in co-substituted BiFeO3 thin films, Applied Physics Letters 105 (17) (2014) 172904. doi:10.1063/1.4900755.

[163] L. Fang, L. You, Y. Zhou, P. Ren, Z. S. Lim, J. Wang, Switchable photovoltaic response from polarization modulated interfaces in $\mathrm{BiFeO} 3$ thin films, Applied Physics Letters 104 (14) (2014) 142903.

[164] P. M. Jones, S. Dunn, Photo-reduction of silver salts on highly heterogeneous lead zirconate titanate, Nanotechnology 18 (2007) 185702.

[165] D. Tiwari, S. Dunn, Photochemistry on a polarisable semi-conductor: what do we understand today?, J Mater Sci 44 (19) (2009) 5063-5079.

[166] A. Haussmann, P. Milde, C. Erler, L. M. Eng, Ferroelectric Lithography: Bottom-up Assembly and Electrical Performance of a Single Metallic Nanowire, Nano Lett. 9 (2) (2009) 763-768.

[167] S. Dunn, D. Tiwari, P. M. Jones, D. E. Gallardo, Insights into the relationship between inherent materials properties of PZT and photochemistry for the development of nanostructured silver, Journal of Materials Chemistry 17 (42) (2007) 4460.

[168] Z. Shen, G. Chen, Z. Chen, X. Qu, Y. Chen, R. Liu, Spatially Selective Photochemical Reduction of Silver on Nanoembossed Ferroelectric PZT Nanowires, Langmuir 27 (9) (2011) 5167-5170.

[169] Y. Sun, B. S. Eller, R. J. Nemanich, Photo-induced Ag deposition on periodically poled lithium niobate: Concentration and intensity dependence, Journal of Applied Physics 110 (8) (2011) 084303. 
[170] S. V. Didziulis, K. D. Butcher, S. L. Cohen, E. I. Solomon, Chemistry of copper overlayers on zinc oxide single-crystal surfaces: model active sites for copper/zinc oxide methanol synthesis catalysts, J. Am. Chem. Soc. 111 (18) (1989) 7110-7123.

[171] S. Roberts, R. J. Gorte, A comparison of Pt overlayers on $\alpha$-Al2o3(0001), $\mathrm{ZnO}(0001) \mathrm{Zn}$, and $\mathrm{ZnO}(000 \overline{1}) \mathrm{O}$, The Journal of Chemical Physics 93 (7) (1990) 5337-5344.

[172] P. Bera, J. M. Vohs, Growth and structure of Pd films on $\mathrm{ZnO}(0001)$, J Chem Phys 125 (16) (2006) 164713.

[173] M. P. Hyman, V. M. Lebarbier, Y. Wang, A. K. Datye, J. M. Vohs, A Comparison of the Reactivity of $\mathrm{Pd}$ Supported on $\mathrm{ZnO}(101 ? 0)$ and $\mathrm{ZnO}(0001)$, J. Phys. Chem. C 113 (17) (2009) 7251-7259.

[174] A. M. Kolpak, I. Grinberg, A. M. Rappe, Polarization Effects on the Surface Chemistry of $\mathrm{PbTiO}_{3}$-Supported Pt Films, Phys. Rev. Lett. 98 (16) (2007) 166101.

[175] H. Falsig, T. Bligaard, J. Rass-Hansen, A. L. Kustov, C. H. Christensen, J. K. Norskov, Trends in catalytic NO decomposition over transition metal surfaces, Top Catal 45 (1-4) (2007) 117-120.

[176] G. Jones, J. G. Jakobsen, S. S. Shim, J. Kleis, M. P. Andersson, J. Rossmeisl, F. Abild-Pedersen, T. Bligaard, S. Helveg, B. Hinnemann, J. R. Rostrup-Nielsen, I. Chorkendorff, J. Sehested, J. K. Norskov, First principles calculations and experimental insight into methane steam reforming over transition metal catalysts, Journal of Catalysis 259 (1) (2008) 147160.

[177] J. Greeley, I. E. L. Stephens, A. S. Bondarenko, T. P. Johansson, H. A. Hansen, T. F. Jaramillo, J. Rossmeisl, I. Chorkendorff, J. K. Norskov, Alloys of platinum and early transition metals as oxygen reduction electrocatalysts, Nat Chem 1 (7) (2009) 552-556.

[178] A. Vojvodic, A. J. Medford, F. Studt, F. Abild-Pedersen, T. S. Khan, T. Bligaard, J. K. Norskov, Exploring the limits: A low-pressure, lowtemperature Haber-Bosch process, Chemical Physics Letters 598 (2014) $108-112$.

[179] A. J. Medford, A. Vojvodic, J. S. Hummelshoj, J. Voss, F. Abild-Pedersen, F. Studt, T. Bligaard, A. Nilsson, J. K. Norskov, From the Sabatier principle to a predictive theory of transition-metal heterogeneous catalysis, Journal of Catalysis 328 (2015) 36-42.

[180] Y. Yun, N. Pilet, U. D. Schwarz, E. I. Altman, Comparison of the interaction of Pd with positively and negatively poled LiNbO3(0 00 1), Surface Science 603 (20) (2009) 3145-3154. 
[181] M. H. Zhao, D. A. Bonnell, J. M. Vohs, Ferroelectric polarization dependent interactions at $\mathrm{Pd}-\mathrm{LiNbO} 3(0001)$ interfaces, Journal of Vacuum Science \& Technology A 27 (6) (2009) 1337-1342.

[182] Y. Cui, J. Briscoe, S. Dunn, Effect of Ferroelectricity on Solar-LightDriven Photocatalytic Activity of BaTiO3 - Influence on the Carrier Separation and Stern Layer Formation, Chem. Mater. 25 (21) (2013) 42154223 .

[183] R. Su, Y. Shen, L. Li, D. Zhang, G. Yang, C. Gao, Y. Yang, SilverModified Nanosized Ferroelectrics as a Novel Photocatalyst, Small 11 (2) (2015) 202-207.

[184] T. Zhao, A. Scholl, F. Zavaliche, K. Lee, M. Barry, A. Doran, M. P. Cruz, Y. H. Chu, C. Ederer, N. A. Spaldin, R. R. Das, D. M. Kim, S. H. Baek, C. B. Eom, R. Ramesh, Electrical control of antiferromagnetic domains in multiferroic BiFeO3 films at room temperature, Nat Mater 5 (10) (2006) 823-829.

[185] J.-G. Park, M. D. Le, J. Jeong, S. Lee, Structure and spin dynamics of multiferroic $\mathrm{BiFeO}_{3}$, Journal of Physics: Condensed Matter 26 (2014) 433202 .

[186] S. K. Srivastav, N. S. Gajbhiye, Low Temperature Synthesis, Structural, Optical and Magnetic Properties of Bismuth Ferrite Nanoparticles, J. Am. Ceram. Soc. 95 (11) (2012) 3678-3682.

[187] S. Li, Y.-H. Lin, B.-P. Zhang, Y. Wang, C.-W. Nan, Controlled Fabrication of BiFeO3 Uniform Microcrystals and Their Magnetic and Photocatalytic Behaviors, J. Phys. Chem. C 114 (7) (2010) 2903-2908.

[188] W. Ji, K. Yao, Y. C. Liang, Bulk Photovoltaic Effect at Visible Wavelength in Epitaxial Ferroelectric BiFeO3 Thin Films, Adv. Mater. 22 (15) (2010) 1763-1766.

[189] P. Kanhere, Z. Chen, A Review on Visible Light Active Perovskite-Based Photocatalysts, Molecules 19 (12) (2014) 19995-20022.

[190] X. Zhang, B. Wang, X. Wang, X. Xiao, Z. Dai, W. Wu, J. Zheng, F. Ren, C. Jiang, Preparation of $\mathrm{M} @ B$ BiFeO3 Nanocomposites $(\mathrm{M}=\mathrm{Ag}, \mathrm{Au})$ Bowl Arrays with Enhanced Visible Light Photocatalytic Activity, J. Am. Ceram. Soc. 98 (7) (2015) 2255-2263.

[191] S. Li, J. Zhang, M. G. Kibria, Z. Mi, M. Chaker, D. Ma, R. Nechache, F. Rosei, Remarkably enhanced photocatalytic activity of laser ablated Au nanoparticle decorated $\mathrm{BiFeO} 3$ nanowires under visible-light, Chemical Communications 49 (52) (2013) 5856.

[192] Molecular beam epitaxy 500. 
[193] S. A. Chambers, Epitaxial growth and properties of thin film oxides, Surface Science Reports 39 (5-6) (2000) 105-180.

[194] C. H. Ahn, K. M. Rabe, J.-M. Triscone, Ferroelectricity at the Nanoscale: Local Polarization in Oxide Thin Films and Heterostructures, Science 303 (5657) (2004) 488-491.

[195] S. M. George, Atomic Layer Deposition: An Overview, Chem. Rev. 110 (1) (2010) 111-131.

[196] M. Knez, K. Nielsch, L. Niinisto, Synthesis and Surface Engineering of Complex Nanostructures by Atomic Layer Deposition, Adv. Mater. 19 (21) (2007) 3425-3438.

[197] X. Wang, S. M. Tabakman, H. Dai, Atomic Layer Deposition of Metal Oxides on Pristine and Functionalized Graphene, J. Am. Chem. Soc. 130 (26) (2008) 8152-8153.

[198] M. Leskela, M. Ritala, Atomic layer deposition chemistry: recent developments and future challenges, Angew. Chem. Int. Ed. Engl. 42 (45) (2003) 5548-5554. doi:10.1002/anie.200301652.

[199] C. H. Ahn, R. H. Hammond, T. H. Geballe, M. R. Beasley, J.-M. Triscone, M. Decroux, O. Fischer, L. Antognazza, K. Char, Ferroelectric field effect in ultrathin SrRuO3 films, Applied Physics Letters 70 (2) (1997) 206-208.

[200] C. H. Ahn, J.-M. Triscone, N. Archibald, M. Decroux, R. H. Hammond, T. H. Geballe, O. Fischer, M. R. Beasley, Ferroelectric Field Effect in Epitaxial Thin Film Oxide $\mathrm{SrCuO} 2 / \mathrm{Pb}(\mathrm{Zr0} 0.52$ ti0.48)O3 Heterostructures, Science 269 (5222) (1995) 373-376.

[201] Y. Zhang, A. Kolmakov, S. Chretien, H. Metiu, M. Moskovits, Control of Catalytic Reactions at the Surface of a Metal Oxide Nanowire by Manipulating Electron Density Inside It, Nano Lett. 4 (3) (2004) 403-407.

[202] Y. Zhang, A. Kolmakov, Y. Lilach, M. Moskovits, Electronic Control of Chemistry and Catalysis at the Surface of an Individual Tin Oxide Nanowire, J. Phys. Chem. B 109 (5) (2005) 1923-1929.

[203] T. Miyasako, M. Senoo, E. Tokumitsu, Ferroelectric-gate thin-film transistors using indium-tin-oxide channel with large charge controllability, Applied Physics Letters 86 (16) (2005) 162902.

[204] Y. Arimoto, H. Ishiwara, Current Status of Ferroelectric Random-Access Memory, MRS Bulletin 29 (11) (2004) 823-828.

[205] Y.-Y. Lin, Y. Zhang, J. Singh, R. York, U. Mishra, Study of charge control and gate tunneling in a ferroelectric-oxide-silicon field effect transistor: Comparison with a conventional metal-oxide-silicon structure, Journal of Applied Physics 89 (3) (2001) 1856-1860. 
[206] C. Song, Global challenges and strategies for control, conversion and utilization of $\mathrm{CO} 2$ for sustainable development involving energy, catalysis, adsorption and chemical processing, Catalysis Today 115 (1-4) (2006) 232 .

[207] K. Sumida, D. L. Rogow, J. A. Mason, T. M. McDonald, E. D. Bloch, Z. R. Herm, T.-H. Bae, J. R. Long, Carbon Dioxide Capture in Metal-Organic Frameworks, Chem. Rev. 112 (2012) 724-781.

[208] E. Hollmann, J. Schubert, R. Kutzner, R. Wordenweber, Stress generated modifications of epitaxial ferroelectric SrTiO3 films on sapphire, Journal of Applied Physics 105 (11) (2009) 114104.

[209] J. H. Haeni, P. Irvin, W. Chang, R. Uecker, P. Reiche, Y. L. Li, S. Choudhury, W. Tian, M. E. Hawley, B. Craigo, A. K. Tagantsev, X. Q. Pan, S. K. Streiffer, L. Q. Chen, S. W. Kirchoefer, J. Levy, D. G. Schlom, Room-temperature ferroelectricity in strained SrTiO3, Nature 430 (7001) (2004) $758-761$.

[210] D. G. Schlom, L.-Q. Chen, C.-B. Eom, K. M. Rabe, S. K. Streiffer, J.-M. Triscone, Strain Tuning of Ferroelectric Thin Films, Annual Review of Materials Research 37 (1) (2007) 589-626.

[211] Y. Inoue, K. Sato, O. Hayashi, Adsorptive properties of semiconducting thin $\mathrm{NiO}$ and $\mathrm{TiO} 2$ films combined with an oppositely polarized ferroelectric support, J. Chem. Soc., Faraday Trans. 183 (9) (1987) 3061-3068.

[212] S. V. Kalinin, B. J. Rodriguez, S. Jesse, E. Karapetian, B. Mirman, E. A. Eliseev, A. N. Morozovska, Nanoscale Electromechanics of Ferroelectric and Biological Systems: A New Dimension in Scanning Probe Microscopy, Annual Review of Materials Research 37 (1) (2007) 189-238.

[213] Y. Inoue, Effects of acoustic waves-induced dynamic lattice distortion on catalytic and adsorptive properties of metal, alloy and metal oxide surfaces, Surface Science Reports 62 (8) (2007) 305-336.

[214] H. Nishiyama, N. Saito, H. Chou, K. Sato, Y. Inoue, Effects of surface acoustic waves on adsorptive properties of $\mathrm{ZnO}$ and $\mathrm{NiO}$ thin films deposited on ferroelectric substrates, Surface Science 433-435 (1999) 525528.

[215] N. Saito, H. Nishiyama, Y. Inoue, Acoustic wave effects on catalysis: design of surfaces with artificially controllable functions for chemical reactions, Applied Surface Science 169-170 (2001) 259-263.

[216] N. V. Burbure, P. A. Salvador, G. S. Rohrer, Photochemical Reactivity of Titania Films on BaTiO3 Substrates: Origin of Spatial Selectivity, Chem. Mater. 22 (21) (2010) 5823-5830. 
[217] N. V. Burbure, P. A. Salvador, G. S. Rohrer, Photochemical Reactivity of Titania Films on BaTiO3 Substrates: Influence of Titania Phase and Orientation, Chem. Mater. 22 (21) (2010) 5831-5837.

[218] Y. Zhang, A. M. Schultz, P. A. Salvador, G. S. Rohrer, Spatially selective visible light photocatalytic activity of $\mathrm{TiO} 2 / \mathrm{BiFeO} 3$ heterostructures, Journal of Materials Chemistry 21 (12) (2011) 4168.

[219] L. Li, G. S. Rohrer, P. A. Salvador, Heterostructured Ceramic Powders for Photocatalytic Hydrogen Production: Nanostructured TiO2 Shells Surrounding Microcrystalline (Ba,Sr)TiO3 Cores, J. Am. Ceram. Soc. 95 (4) (2012) 1414-1420.

[220] L. Li, Y. Zhang, A. M. Schultz, X. Liu, P. A. Salvador, G. S. Rohrer, Visible light photochemical activity of heterostructured PbTiO3-TiO2 core-shell particles, Catalysis Science \& Technology 2 (9) (2012) 1945.

[221] J. H. Lee, A. Selloni, $\mathrm{TiO}_{2} /$ Ferroelectric Heterostructures as Dynamic Polarization-Promoted Catalysts for Photochemical and Electrochemical Oxidation of Water, Phys. Rev. Lett. 112 (19) (2014) 196102.

[222] J. Greeley, T. F. Jaramillo, J. Bonde, I. Chorkendorff, J. K. Norskov, Computational high-throughput screening of electrocatalytic materials for hydrogen evolution, Nat Mater 5 (11) (2006) 909-913.

[223] J. Greeley, J. K. Norskov, M. Mavrikakis, Electronic Structure and Catalysis on Metal Surfaces, Annual Review of Physical Chemistry 53 (1) (2002) 319-348.

[224] M. P. Andersson, T. Bligaard, A. Kustov, K. E. Larsen, J. Greeley, T. Johannessen, C. H. Christensen, J. K. Norskov, Toward computational screening in heterogeneous catalysis: Pareto-optimal methanation catalysts, Journal of Catalysis 239 (2) (2006) 501-506.

[225] A. Vojvodic, J. K. Norskov, Optimizing Perovskites for the WaterSplitting Reaction, Science 334 (6061) (2011) 1355-1356.

[226] M. V. Twigg, Progress and future challenges in controlling automotive exhaust gas emissions, Applied Catalysis B: Environmental 70 (1-4) (2007) $2-15$.

[227] P. Granger, V. I. Parvulescu, Catalytic NOx Abatement Systems for Mobile Sources: From Three-Way to Lean Burn after-Treatment Technologies, Chem. Rev. 111 (5) (2011) 3155-3207.

[228] S. Matsumoto, Recent advances in automobile exhaust catalysts, Catalysis Today 90 (3-4) (2004) 183-190. 
[229] U. Deka, I. Lezcano-Gonzalez, B. M. Weckhuysen, A. M. Beale, Local Environment and Nature of $\mathrm{Cu}$ Active Sites in Zeolite-Based Catalysts for the Selective Catalytic Reduction of NOx, ACS Catal. 3 (3) (2013) $413-427$.

[230] A. M. Beale, F. Gao, I. Lezcano-Gonzalez, C. H. F. Peden, J. Szanyi, Recent advances in automotive catalysis for $\mathrm{NO}_{\mathrm{x}}$ emission control by small-pore microporous materials, Chem. Soc. Rev.

[231] B. Hammer, J. K. Norskov, Theoretical surface science and catalysis - calculations and concepts, Vol. 45 of Impact of Surface Science on Catalysis, Academic Press, 2000, pp. 71-129.

[232] M. Mavrikakis, B. Hammer, J. K. Norskov, Effect of Strain on the Reactivity of Metal Surfaces, Phys. Rev. Lett. 81 (13) (1998) 2819-2822.

[233] V. Stamenkovic, B. S. Mun, K. J. J. Mayrhofer, P. N. Ross, N. M. Markovic, J. Rossmeisl, J. Greeley, J. K. Norskov, Changing the Activity of Electrocatalysts for Oxygen Reduction by Tuning the Surface Electronic Structure, Angewandte Chemie 118 (18) (2006) 2963-2967.

[234] K. Garrity, A. M. Kolpak, S. Ismail-Beigi, E. I. Altman, Chemistry of Ferroelectric Surfaces, Adv. Mater. 22 (26-27) (2010) 2969-2973.

[235] F. Studt, F. Abild-Pedersen, T. Bligaard, R. Z. Sorensen, C. H. Christensen, J. K. Norskov, Identification of Non-Precious Metal Alloy Catalysts for Selective Hydrogenation of Acetylene, Science 320 (5881) (2008) $1320-1322$.

[236] P. Ferrin, D. Simonetti, S. Kandoi, E. Kunkes, J. A. Dumesic, J. K. Norskov, M. Mavrikakis, Modeling Ethanol Decomposition on Transition Metals: A Combined Application of Scaling and Bronsted-Evans-Polanyi Relations, J. Am. Chem. Soc. 131 (16) (2009) 5809-5815.

[237] J. W. Erisman, M. A. Sutton, J. Galloway, Z. Klimont, W. Winiwarter, How a century of ammonia synthesis changed the world, Nature Geosci 1 (10) (2008) 636-639.

[238] A. Hellman, K. Honkala, I. N. Remediakis, A. Logadottir, A. Carlsson, S. Dahl, C. H. Christensen, J. K. Norskov, Insights into ammonia synthesis from first-principles, Surface Science 600 (18) (2006) 4264-4268.

[239] A. Logadottir, T. H. Rod, J. K. Norskov, B. Hammer, S. Dahl, C. J. H. Jacobsen, The Brønsted-Evans-Polanyi Relation and the Volcano Plot for Ammonia Synthesis over Transition Metal Catalysts, Journal of Catalysis 197 (2) (2001) 229-231.

[240] A. Vojvodic, A. Hellman, C. Ruberto, B. I. Lundqvist, From Electronic Structure to Catalytic Activity: A Single Descriptor for Adsorption and Reactivity on Transition-Metal Carbides, Phys. Rev. Lett. 103 (14) (2009) 146103. 
[241] I. C. Man, H.-Y. Su, F. Calle-Vallejo, H. A. Hansen, J. I. Martinez, N. G. Inoglu, J. Kitchin, T. F. Jaramillo, J. K. Norskov, J. Rossmeisl, Universality in Oxygen Evolution Electrocatalysis on Oxide Surfaces, ChemCatChem 3 (7) (2011) 1159-1165.

[242] A. Vojvodic, J. K. Norskov, F. Abild-Pedersen, Electronic Structure Effects in Transition Metal Surface Chemistry, Top Catal 57 (1-4) (2013) $25-32$.

[243] S. Linic, J. Jankowiak, M. A. Barteau, Selectivity driven design of bimetallic ethylene epoxidation catalysts from first principles, Journal of Catalysis 224 (2) (2004) 489-493.

[244] J. K. Norskov, F. Abild-Pedersen, F. Studt, T. Bligaard, Density functional theory in surface chemistry and catalysis, PNAS 108 (3) (2011) 937-943.

[245] C. J. H. Jacobsen, S. Dahl, B. S. Clausen, S. Bahn, A. Logadottir, J. K. Norskov, Catalyst Design by Interpolation in the Periodic Table: Bimetallic Ammonia Synthesis Catalysts, J. Am. Chem. Soc. 123 (34) (2001) 8404-8405.

[246] F. Besenbacher, I. Chorkendorff, B. S. Clausen, B. Hammer, A. M. Molenbroek, J. K. Norskov, I. Stensgaard, Design of a Surface Alloy Catalyst for Steam Reforming, Science 279 (5358) (1998) 1913-1915.

[247] J. H. Montoya, C. Tsai, A. Vojvodic, J. K. Norskov, The Challenge of Electrochemical Ammonia Synthesis: A New Perspective on the Role of Nitrogen Scaling Relations, ChemSusChem 8 (13) (2015) 2180-2186.

[248] F. Abild-Pedersen, J. Greeley, F. Studt, J. Rossmeisl, T. R. Munter, P. G. Moses, E. Skulason, T. Bligaard, J. K. Norskov, Scaling Properties of Adsorption Energies for Hydrogen-Containing Molecules on TransitionMetal Surfaces, Phys. Rev. Lett. 99 (1) (2007) 016105.

[249] G. Jones, T. Bligaard, F. Abild-Pedersen, J. K. Norskov, Using scaling relations to understand trends in the catalytic activity of transition metals, Journal of Physics: Condensed Matter 20 (2008) 064239.

[250] L. C. Grabow, F. Studt, F. Abild-Pedersen, V. Petzold, J. Kleis, T. Bligaard, J. K. Norskov, Descriptor-Based Analysis Applied to HCN Synthesis from NH3 and CH4, Angew. Chem. Int. Ed. 50 (20) (2011) 4601-4605.

[251] A. Vojvodic, J. K. Norskov, New Design Paradigm for Heterogeneous Catalysts, Natl Sci Rev (2015) nwv023.

[252] J. H. Lunsford, Catalytic conversion of methane to more useful chemicals and fuels: a challenge for the 21 st century, Catalysis Today 63 (2-4) (2000) $165-174$. 
[253] P. V. L. Reddy, K.-H. Kim, H. Song, Emerging green chemical technologies for the conversion of $\mathrm{CH} 4$ to value added products, Renewable and Sustainable Energy Reviews 24 (2013) 578-585.

[254] O. Benlounes, S. Mansouri, C. Rabia, S. Hocine, Direct oxidation of methane to oxygenates over heteropolyanions, Journal of Natural Gas Chemistry 17 (3) (2008) 309-312.

[255] S. Murcia-Lopez, K. Villa, T. Andreu, J. R. Morante, Partial Oxidation of Methane to Methanol Using Bismuth-Based Photocatalysts, ACS Catal. 4 (9) (2014) 3013-3019.

[256] E. Jobson, Future Challenges in Automotive Emission Control, Topics in Catalysis 28 (1-4) (2004) 191-199.

[257] S.-W. Cheong, M. Mostovoy, Multiferroics: a magnetic twist for ferroelectricity, Nat Mater 6 (1) (2007) 13-20.

[258] T. Kimura, T. Goto, H. Shintani, K. Ishizaka, T. Arima, Y. Tokura, Magnetic control of ferroelectric polarization, Nature 426 (6962) (2003) $55-58$.

[259] Y. Yamasaki, S. Miyasaka, Y. Kaneko, J.-P. He, T. Arima, Y. Tokura, Magnetic Reversal of the Ferroelectric Polarization in a Multiferroic Spinel Oxide, Phys. Rev. Lett. 96 (20) (2006) 207204.

[260] N. Hur, S. Park, P. A. Sharma, J. S. Ahn, S. Guha, S.-W. Cheong, Electric polarization reversal and memory in a multiferroic material induced by magnetic fields, Nature 429 (6990) (2004) 392-395.

[261] A. K. Zvezdin, A. M. Kadomtseva, S. S. Krotov, A. P. Pyatakov, Y. F. Popov, G. P. Vorobev, Magnetoelectric interaction and magnetic field control of electric polarization in multiferroics, Journal of Magnetism and Magnetic Materials 300 (1) (2006) 224-228.

[262] A. Kumar, G. L. Sharma, R. S. Katiyar, R. Pirc, R. Blinc, J. F. Scott, Magnetic control of large room-temperature polarization, Journal of Physics: Condensed Matter 21 (2009) 382204. 


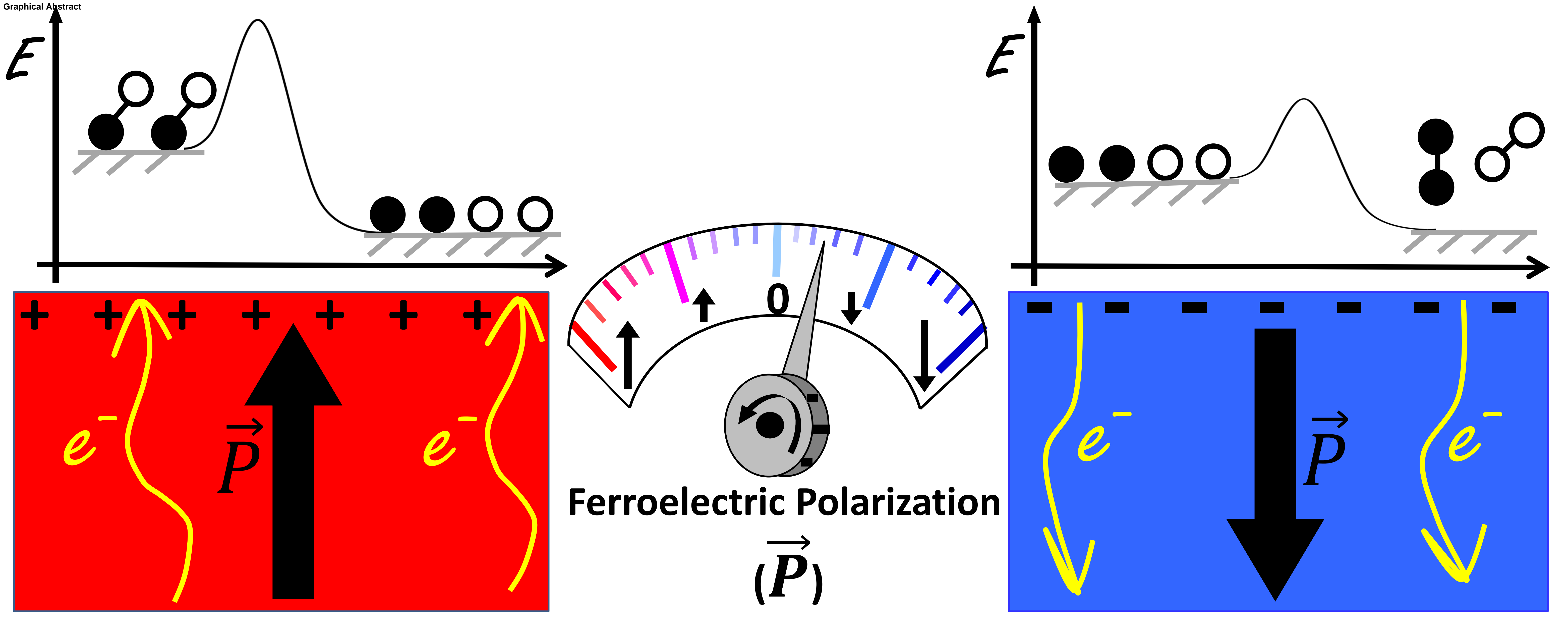

